\title{
Three-dimensional imaging of swirled spray injection in a generic aero engine burner under realistic operating conditions
}

\author{
Joachim Klinner ${ }^{1}\left[\right.$ C Christian E. Willert ${ }^{1}$ \\ Received: 4 March 2021 / Revised: 1 October 2021 / Accepted: 6 November 2021 / Published online: 1 December 2021 \\ (c) The Author(s) 2021
}

\begin{abstract}
Tomographic shadowgraph imaging is applied to reconstruct the instantaneous three-dimensional spray field immediately downstream of a generic aero engine fuel injector. Within the swirl passage of the injector model, a single kerosene jet undergoes air-blast atomization in a cross-flow configuration at Weber numbers of We $=360-770$, air pressures of $p_{a}=4-7$ bar and air temperatures of $T_{a}=440-570 \mathrm{~K}$. High-speed, high magnification shadowgraphy is used to visualize the initial fuel atomization stages within the fuel injector before the spray enters the spray chamber. The 4-camera tomographic measurement setup is described in detail and includes a depth-of-field analysis with respect to droplet size based on Mie simulations and calibration data of the point-spread function. For a volume size of $16 \times 13 \times 10 \mathrm{~mm}^{3}$, the smallest resolvable droplet diameter is estimated to be $d=10 \mu \mathrm{m}$ within the focal plane and increases to $d \approx 20 \mu \mathrm{m}$ toward the edges of the volume. Droplet velocities above the resolution limit were retrieved by $3-\mathrm{d}$ cross-correlation of two volumetric reconstructions recorded at two consecutive time-steps. This is accompanied by an error analysis on the random error dependency on the camera viewing geometry. The results indicate increasing motion and fluctuations of the spray tail with increasing temperature and Weber number. Validation against PDA data further downstream of the burner plate revealed consistency for size classes $d=10 \mu \mathrm{m}$ and $d=15 \mu \mathrm{m}$. Deviations from PDA occur in regions with strong velocity gradients due to different spatial resolutions, the presence of reconstruction ambiguities (ghost particles), uncertainties inherent to the two-frame cross-correlation of spray volumes and the finite LED pulse duration.
\end{abstract}

Joachim Klinner

joachim.klinner@dlr.de

1 German Aerospace Center (DLR), Institute of Propulsion

Technology, Engine Measurement Systems, Linder Hoehe,

Koeln D-51147, Germany 


\section{Graphical Abstract}
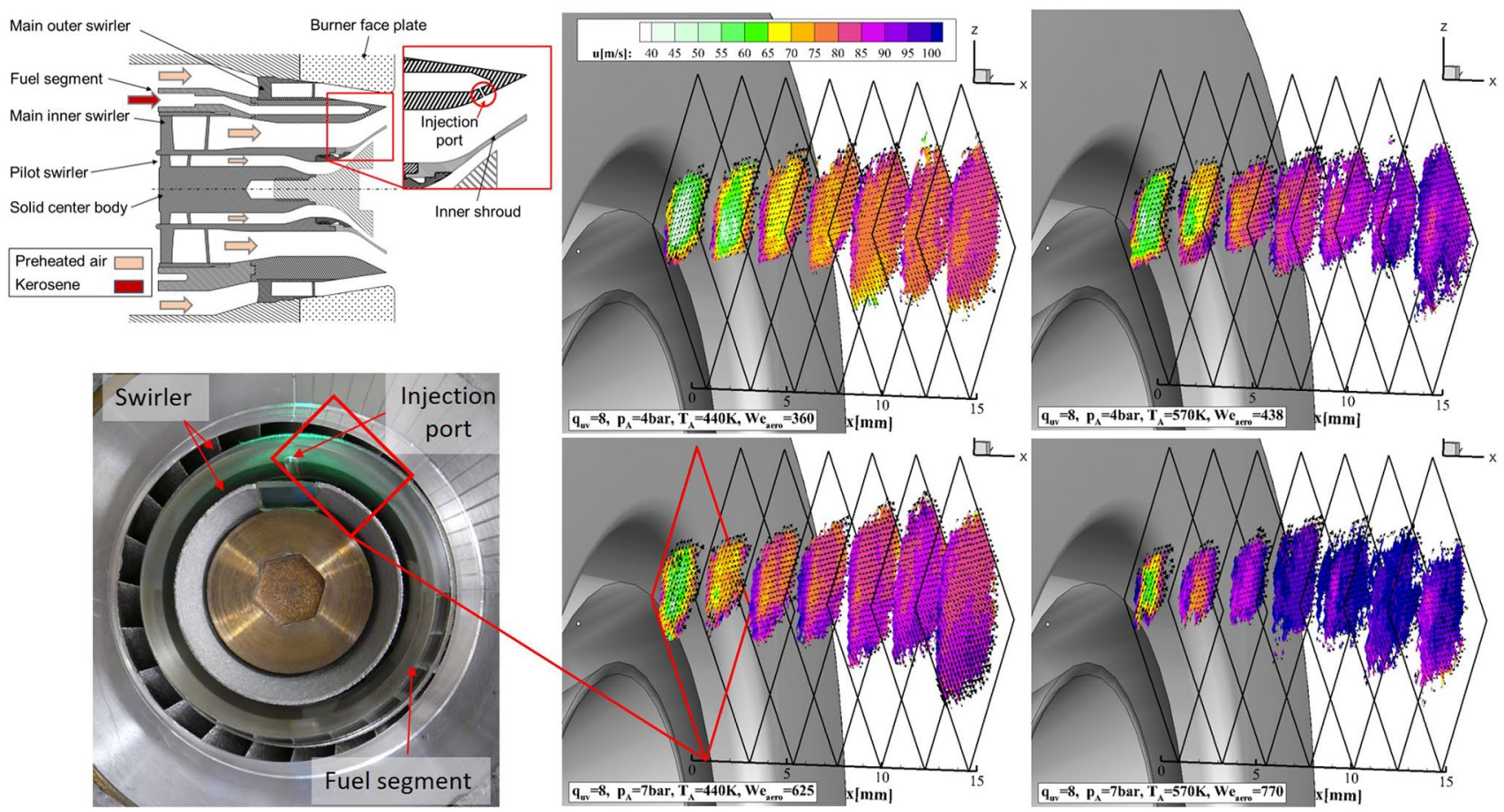

\section{Introduction}

The optimization of aero engine combustors requires a detailed knowledge of the fuel atomization process including fuel placement, breakup length scales, spray penetration depth, droplet sizes and velocities. The acquisition of relevant experimental data on kerosene atomization on the other hand raises significant challenges such as providing realistic operating conditions and sufficient optical access. Another obstacle is that the dispersion of liquid kerosene by aero engine fuel injectors is driven by a highly threedimensional, typically swirling flow. Frequently, air-blast atomization of liquid kerosene films or jets is applied. The jet or film breakup in itself already is a highly complex process and subject of a large body of literature. Improved insight into the subject strongly relies on (optical) diagnostic methods to provide validation data for theoretical and numerical modelling (Fansler and Parrish 2015). The present effort is aimed at capturing the spray characteristics in an engine relevant configuration involving elevated pressures (4-7 bar) and preheated air with nozzle exit velocities in the $50-100 \mathrm{~m} / \mathrm{s}$ range. Aside from the visualization of the initial spray column at its point of injection, the instantaneous three-dimensional placement of atomized fuel within the combustion volume is mapped both spatially and temporally.
With regard to three-dimensional spray imaging, considerable work is documented in the field of holographic imaging. Among the early works, Jones et al. (1978) report on film-based inline holography to infer droplet position and size. (Kang and Poulikakos 1996) used a double reference beam holographic setup with two pulses staggered in time to determine droplet size and velocity in an impinging jet spray. Focused image holography with side lighting was used to image the complex ligament formation near the spray nozzle exit (Santangelo and Sojka 1994). Multiple exposure recordings obtained with a similar inline setup were used to recover three-dimensional droplet velocities in sprays (Lü et al. 2009; Yang and Kang 2009). Inline holographic approaches perform well for sparsely populated droplet volumes but suffer from strong reference beam attenuation and speckle noise in high droplet density regions. In addition, the limited angular aperture of digital inline holograms results in ellipsoidal and elongated reconstructions of spherical particles (Meng and Hussain 1995). This effect can be reduced by combining the inline reference beam with a side scattered object wave which improves the contrast of the recorded diffraction patterns and therefore increases axial resolution (Cao et al. 2008) or by crossing several inline holographic setups (Soria and Atkinson 2008). With the increased availability of high-resolution, high-speed imaging the framing 
rate of digital holography could be increased to temporally resolve the liquid atomization process well into the $\mathrm{kHz}$ range (Guildenbecher et al. 2016; Wu et al. 2021).

Whereas holography has been used quite extensively for the investigation of sprays, the literature reports only few applications of tomography for three-dimensional spray reconstruction. An ultrafast X-ray tomography technique was used by Cai et al. (2003) and Liu et al. (2009) for the transient, near-field spray characterization of an multi-hole injector at frame rates of about $48 \mathrm{kHz}$. Tomographic reconstruction of the instantaneous, phase-averaged spray distribution was achieved through rotation $\left(\approx 1^{\circ}\right.$ steps on $\left.180^{\circ}\right)$ and vertical translation of the injector in the X-ray imaging path. Compared to optical imaging, $\mathrm{X}$-ray imaging has the advantage that the recorded radiation intensity mainly depends upon the absorption in the liquid, since scattering can be neglected at small wavelengths. Thus, quantitative measurements of the local fuel mass fraction become feasible (Halls et al. 2013; Coletti et al. 2014). The above mentioned tomographic techniques rely on sequential recording of several projections and thus are limited to providing timeor phase-averaged data. Using a triple-path X-ray high-speed imaging setup, Halls et al. (2019) demonstrated the feasibility of capturing the time-evolving 3-d structure of a complex spray undergoing breakup. After tomographic reconstruction of the spray, quantitative measurements of the volume-mass fraction were possible. With this setup, a temporal resolution of $20 \mathrm{kHz}$ with a camera gating of $49 \mu$ s could be achieved.

For the qualitative and quantitative visualization of atomizing sprays, back-light imaging is used very extensively, in part, because of its rather straightforward implementation. The approach essentially relies on the attenuation of light due to the presence of droplets in the optical path between light source and detector (camera) which could best be described as a shadow imaging configuration. While singleaxis imaging configurations only permit a depth-integrated view of the typically three-dimensional spray structures, multiple view imaging arrangements are required to provide volume resolved data. In this regard, "dual-angle" backlight imaging using two orthogonal viewing axes was introduced by Kourmatzis et al. (2017) and later extended with particle tracking velocimetry (PTV) by Pham et al. (2017) to simultaneously provide measurements of droplet volumes and velocity. With only two views, these imaging configurations are suitable for the investigation of single droplets or liquid filaments but face difficulties in denser sprays due to occlusion of droplets. By increasing the number of viewing axes, the reconstruction of denser droplet fields is becomes possible, such as demonstrated by the authors for the reconstruction of the spray field of a hollow cone atomizer (Klinner and Willert 2012). Termed tomographic shadowgraph imaging, the technique relies on the three-dimensional reconstruction of the droplet field from four (or more) back-lit images using algorithms originally developed for tomographic particle image velocimetry (tomo-PIV, Elsinga et al. 2006).

The present contribution describes the adaptation and application of the tomographic shadowgraph imaging technique for the investigation of kerosene spray atomization in a high pressure environment that is representative of realistic aero engine operating conditions. The generic burner employs air-blast atomization of a single jet in swirling cross-flow in the annular main stage. Of particular interest to spray investigation is the near field where the fragments of the kerosene jet leave the annular gap of the burner plate, which cannot be accessed by LDA/PDA. The present application intends to provide insight into the instantaneous spray tail trajectory and the spatial distribution of liquid phase above the resolution limit.

Various aspects concerning the successful adaption of the tomographic shadowgraph imaging setup to the facility are described. After tomographic reconstruction of the droplet fields, droplet velocities are recovered by 3 -d cross-correlation analysis of small interrogation volumes from two consecutive time steps as known from conventional tomographic PIV (Elsinga et al. 2006). Downstream of the burner plate, in a region where spherical drops are expected, exemplary profiles of axial and tangential droplet velocities are compared with PDA measurements.

The following paper first outlines the test facility and its operating conditions. The section on instrumentation describes a shadowgraph imaging setup to capture the kerosene spray at its point of injection within the fuel injector followed by a multi-view shadowgraph setup for 3-d imaging of the atomized spray downstream of the fuel injector within the spray chamber. This is accompanied by an assessment of the optical characteristics regarding depth-of-field and spatial resolution. Details on optical distortion compensation are provided. The results section presents exemplary visualizations and data of the reconstructed spray for different operating conditions and discusses the influence of injection rate and pressure. Where possible the velocity data are compared to PDA measurements obtained for the same operating conditions. The paper concludes with a summary of the primary findings and lessons learned and provides an outlook on possible improvements of the employed measurement techniques.

\section{Test facility, fuel injector model and operation conditions}

Spray measurements are performed in a non-reactive kerosene-air flow in the optical swirling spray injector test rig (OSSI) at the DLR Institute of Propulsion Technology in Cologne. The design of the test rig and the generic fuel 
injector model geometry are described in detail by Freitag (2016, 2018, 2019).

Figure 1 shows a longitudinal section of the test rig. The spray chamber has a length of $200 \mathrm{~mm}$ and a square crosssection of internal width of $102 \mathrm{~mm}$. Pressure windows of $35 \mathrm{~mm}$ thickness and liner windows of $7 \mathrm{~mm}$ thickness provide optical access to the test-section from four sides. Additional cooling air passes through the gap between pressure and liner window to protect the glass from thermal loading and to keep the external pressure casing at ambient temperature levels. The fuel injector model adopts the characteristics of a fuel-staged lean burner and is supplied with preheated and pressurized air through an upstream settling chamber (plenum).

Inside the plenum a baffle with interchangeable screens provides flow conditioning and control of the pressure drop of the fuel injector model. The fuel supply line of the fuel injector passes through the preheated air flow preheating the fuel (Jet-A1 kerosene) prior to injection. The preheating temperatures are provided in Table 1. An exchangeable critical nozzle downstream of the spray chamber builds up pressure and provides mass flow control. For safety and environmental reasons, the fuel-air mixture is fed through a catalytic combustor after leaving the critical nozzle.

The generic aero engine fuel injector model represents that of a lean staged industrial fuel injector. In the current configuration, the pilot stage is replaced by a solid center body (see Fig. 2). The injector model employs air-blast atomization of a single jet in cross-flow in the main stage and contains three annular passages with co-rotating swirl generators: the main outer swirler (MOS), the main inner swirler (MIS) and the passage surrounding the center body (PS). During measurements, the pressure drop across the burner was kept constant at $4 \%$. The total flow-through geometric area of the burner model of $860 \mathrm{~mm}^{2}$ divides among the passages as follows: $44 \%$ MOS, $47 \%$ MIS and

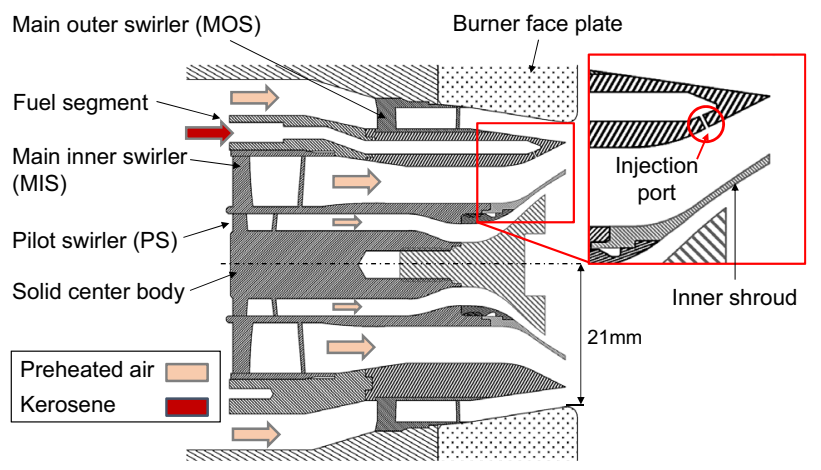

Fig. 2 Aero engine burner model with single fuel injector circled in red (adopted from Freitag 2018)

9\% PS. The effective area, as calculated by scaling the geometric cross-sectional area with the discharge coefficients of each passage, is $596 \mathrm{~mm}^{2}$ (44\% MOS, 46\% MIS, $10 \%$ PS). The latter was verified by LDA measurements which are reproduced together with a more detailed description of the injector model by Freitag $(2018,2019)$.

Jet-A1 fuel is injected through a single bore of $0.88 \mathrm{~mm}$ length and a diameter of $D_{0}=0.29 \mathrm{~mm}\left(\mathrm{~L} / D_{0}=3\right)$. This fuel injection port is located in the conical main module which is placed between the two co-rotating swirl generators. The liquid jet of fuel is injected orthogonal to the conical surface $6 \mathrm{~mm}$ upstream of the fuel injector's exit plane. Strong shear forces of the air flow interact with the liquid jet causing it to fragment into ligaments and droplets that are then carried in swirl direction and leave the annular passage premixed with air.

Table 1 summarizes the operation conditions. During the experiments described herein, the pressure inside the spray chamber $p_{a}$ was varied between 4 bar and 7 bar with air flow preheating ranging between $440 \mathrm{~K} \leq T_{a} \leq 570 \mathrm{~K}$. The static air pressure $p_{a}$ was measured through a port in

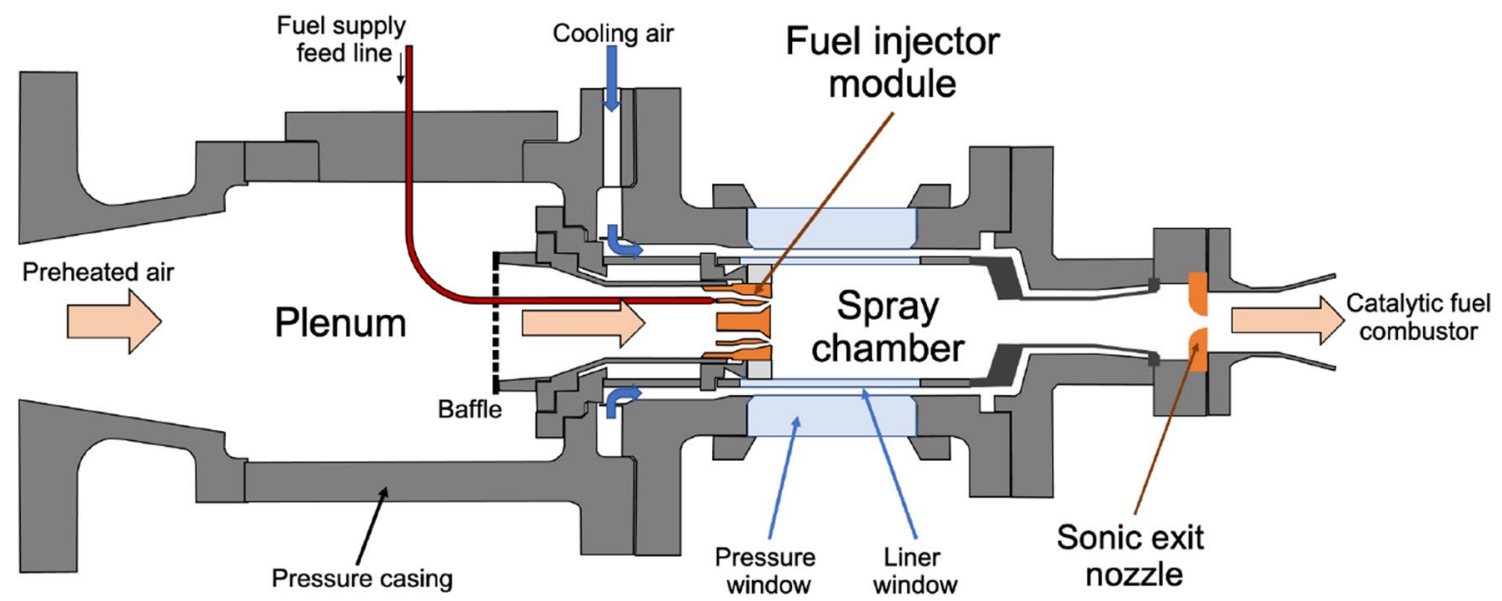

Fig. 1 Schematic of the optical swirling spray injector test rig (cf. Freitag 2018) 
Table 1 Operation conditions of single jet in cross-flow atomization for the fuel injector model used for tomographic shadowgraph imaging. Meaning of subscripts: $\mathrm{a}=\mathrm{air}, \mathrm{k}=$ kerosene

\begin{tabular}{llllllll}
\hline OP no. & $p_{a}[$ bar $]$ & $T_{a}[K]$ & $U_{a}[\mathrm{~m} / s]$ & $T_{k}[K]$ & $\dot{m}_{k}[\mathrm{~g} / s]$ & $q$ \\
\hline 1 & 4 & 440 & 86 & 348 & 0.8 & 360 \\
2 & 4 & 570 & 93 & 422 & 0.7 & 8 \\
3 & 7 & 440 & 86 & 347 & 1.0 & 838 \\
4 & 7 & 570 & 98 & 396 & 1.0 & 625 \\
\hline
\end{tabular}

the liner wall downstream of the liner window and the air temperature $T_{a}$ by a thermocouple between baffle and fuel injector model (see Fig. 1 and Freitag (2018)).

The liquid-to-air momentum flux ratio $q=\rho_{k} U_{k}^{2} / \rho_{a} / U_{a}^{2}$ was kept constant and is calculated from velocities of fuel $U_{k}$ and air $U_{a}$, which are determined by the continuity equation using the kerosene massflow and injection port area, respectively, the burner massflow and the effective split ratios given above to determine the massflow through the MIS. The velocities $U_{a}$ given in Table 1 provide an estimate of the transverse component with respect to the axis of the injection port and were derived from estimates of the axial and radial velocitiy components using the continuity equation and passage geometry while the tangential velocity was neglected (for further details cf. Freitag (2018)). The fuel density $\rho_{k}$ is determined by the fuel temperature measured by a thermocouple about $5 \mathrm{~mm}$ upstream of the injection port while air density $\rho_{a}$ is based on pressure and temperature values measured between baffle and fuel injector. During the investigations presented here, the aerodynamic Weber number We $=\rho_{a} U_{a}^{2} D_{0} / \sigma_{k}$ ranged from 360 to 770 with $D_{0}$ being the diameter of the injection port. Since estimation of the Weber number is based on slightly cooler kerosene temperatures measured about $5 \mathrm{~mm}$ upstream of the injection port, it might be slightly underestimated due to an overestimation of the surface tension of the kerosene.

For the high-speed visualizations of the jet disintegration inside the MIS shroud, a further glass fuel segment made of quartz glass was manufactured in addition to the metallic one. Due to limitations of the manufacturing process of the injection port in quartz glass with ultra-sonic drilling, a larger bore diameter of the injection port had to be accepted. The bore diameter $D_{0}$ of the glass variant was measured optically and, at $0.39 \mathrm{~mm}$, is about $34 \%$ larger than the metallic variant while the length of the bore remains constant. In addition, to make the injector port accessible for HS visualizations, a rectangular quartz window was inserted and glued into the inner shroud (cf. Fig. 3). This window has a length of $4.6 \mathrm{~mm}$ along the shroud deflection and a width of $7.2 \mathrm{~mm}$ in the circumferential direction of the inner shroud. The resulting increase in cross-sectional area within the arc section of the annular gap containing the secant of the rectangular window is on the order of 7\%. Both geometry changes (bore diameter and gap area) were considered when setting the operating conditions for the glass version to achieve the same liquid-to-air momentum flux ratio for both burner models. This ratio essentially determines the trajectory of the kerosene jet and thus also whether the fuel jet impacts the opposite inner shroud. The significantly larger
Fig. 3 Photograph of the fuel injector model with glass fuel segment and window insert with detail showing the injection port rotated to a 7:30 $\mathrm{h}$ position and optical access in inner shroud for shadowgraphy of fuel injection

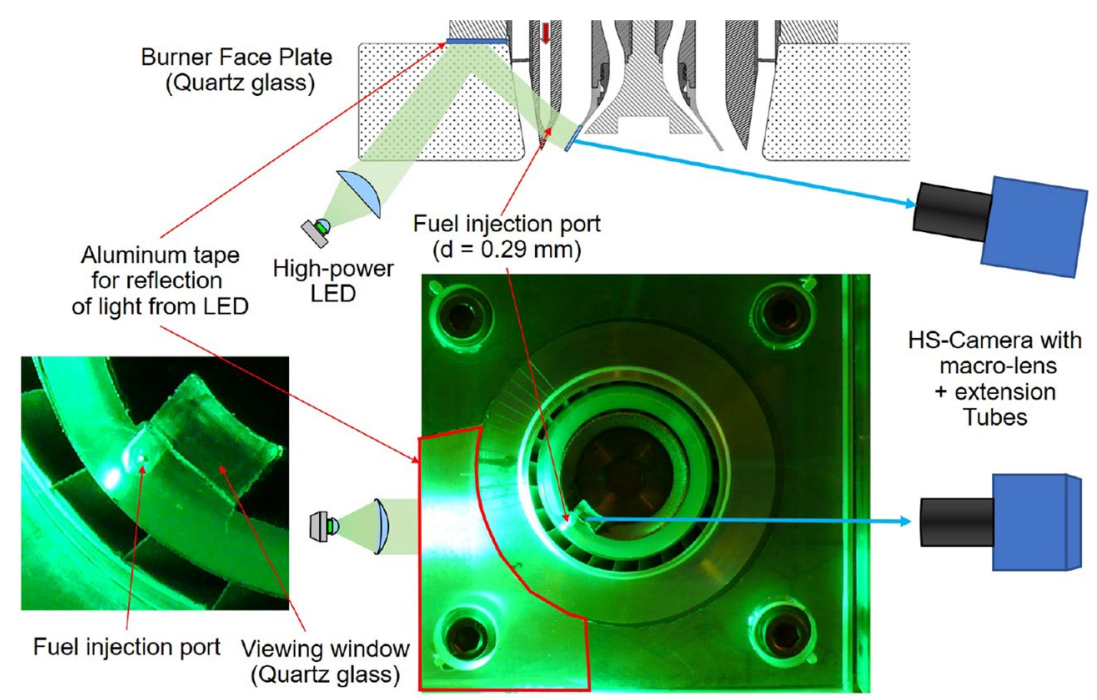


diameter of the injector bore and the enlarged gap area result in approx. $16 \%$ higher Weber numbers for the glass variant of the fuel injector at the same $q$, with slightly lower $U_{a}$ and higher $\dot{m}_{k}$. However, since the high-speed visualizations are intended only for qualitative assessment of the jet disintegration and to prove that the jet does not impact the opposite shroud, the slightly increased Weber numbers of the glass configuration were considered acceptable.

\section{Optical instrumentation}

\subsection{High-speed imaging of the fuel injection}

Shadowgraph imaging of the initial fuel jet within the inner swirl channel was performed both to gain insight into the dynamics of primary atomization of the fuel jet and to adjust the momentum flux ratio $q$ in a way that the exiting jet does not impact onto the opposite $\mathrm{V}$-shroud. To gain visual access to the inner swirl channel, a 0.5 -mm-thin window of $7.2 \times 4.6 \mathrm{~mm}^{2}$ was inserted into the $\mathrm{V}$-shroud. The fuel injection port was rotated to a $7: 30 \mathrm{~h}$ position to obtain an oblique view of the liquid jet within the flow downstream of the inner swirler (cf. Fig. 3). A high-speed camera (Phantom SA5) equipped with a macro-lens (Nikon, Micro Nikkor 200/4) at f-number 8 and extension tubes enabled spray visualizations at a magnification of $12.8 \mu \mathrm{m} / \mathrm{pixel}(M=1.56)$ at a working distance of $\approx 500 \mathrm{~mm}$. Images were recorded at $54 \mathrm{kfps}$ and $75 \mathrm{kfps}$ with corresponding fields of view of $6.6 \times 3.1 \mathrm{~mm}^{2}(512 \times 240$ pixels $)$ and $4.1 \times 3.4 \mathrm{~mm}^{2}$ $(320 \times 264$ pixels $)$. To compensate for optical distortion due to off-normal viewing of $20^{\circ}$ through the 35 -mm-thick pressure window, a compensator glass plate of similar thickness was placed along the observation beam path. By tilting the plate to a similar angle orthogonal to the pressure window the optical distortion could be significantly reduced (for details cf. Sect. 3.3). In order to establish back illumination of the spray, the burner face plate and annular fuel segment were made of quartz glass. Reflector tape placed on the back side of the burner face plate allowed the illumination cone of the LED light source to be redirected into the optical path of the camera as shown in Fig. 3. A green high-power LED (Luminus SST-90-G) pulsed at an effective pulse width of $800 \mathrm{~ns}$ served as the light source. A condenser lens placed in front of the LED maximized the luminance by projecting the light emitting surface onto the glass fuel segment.

\subsection{Tomographic shadowgraph imaging setup}

Photographs of the spray facility with the installed tomographic imaging hardware are provided in Fig. 4 and will be described in detail in the following. The multi-camera setup involves four synchronized scientific CMOS cameras (ILA.sCMOS) with macrolenses (Nikon Nikkor Micro $105 \mathrm{~mm} / 2.8$ or Nikkor Micro $200 \mathrm{~mm} / 4)$. Each camera features an array size of $2560 \times 2160$ pixels at a pitch of $6.5 \mu \mathrm{m}$ per pixel and is capable of acquiring image pairs with sub-microsecond inter-framing times at image-pair acquisition rates up to $25 \mathrm{~Hz}$. The cameras are arranged in a circular arc around the facility as outlined in Fig. 5 using a common coordinate system subscripted with ${ }_{T S}$. Axis $x_{T S}$ is aligned with the burner axis $x$, while the $y_{T S}$ and $z_{T S}$ axes are rotated by $45^{\circ}$ with respect to the facility's $y$ and $z$ axes. With respect to the $x_{T S}-z_{T S}$ plane, the viewing axes of the cameras are $-45^{\circ},-20^{\circ},+25^{\circ}$ and $+45^{\circ}$. Within this coordinate system, the tomographically reconstructed volume has a size of $16 \times 13 \times 10 \mathrm{~mm}^{3}\left(x_{T S} \times y_{T S} \times z_{T S}\right)$ and is located immediately downstream of the annular passage with the injection port located $6 \mathrm{~mm}$ further upstream (outlined in red in Fig. 5). The depth of the volume $\Delta z_{T S}=10 \mathrm{~mm}$ is determined by the depth of field as described Sect. 5.1.

The volume is illuminated using inline illumination for each camera by means of high power green LEDs (Luminus, SST-90, green) whose light was collimated
Fig. 4 Photographs of the optical swirling spray injector test rig with installed multi camera imaging setup; Left camera arrangement; Right detail with calibration target in place
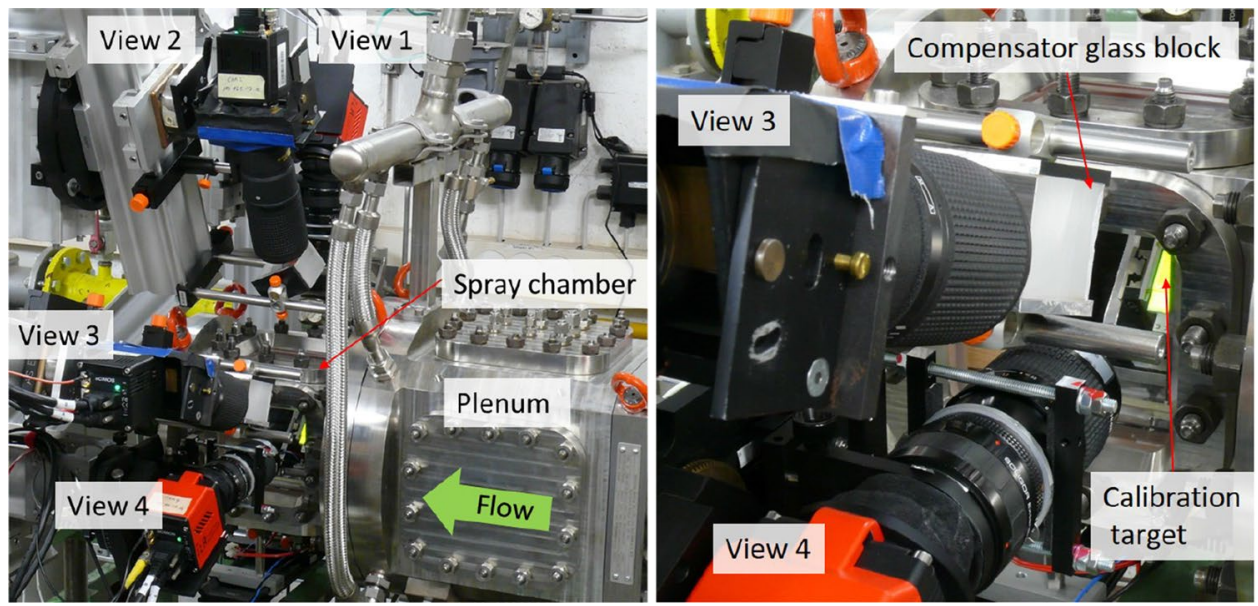
Fig. 5 Top Photograph of the generic fuel injector with a transparent fuel segment (used for spray visualization) and injection port rotated to top center. Bottom Orientation of the measurement volume for 3-d spray imaging; left Fuel injector model with annular passage and injection port; right axial view; The red box indicates the volumetric measurement domain for tomographic data. PDA measurements were performed along the red dotted lines

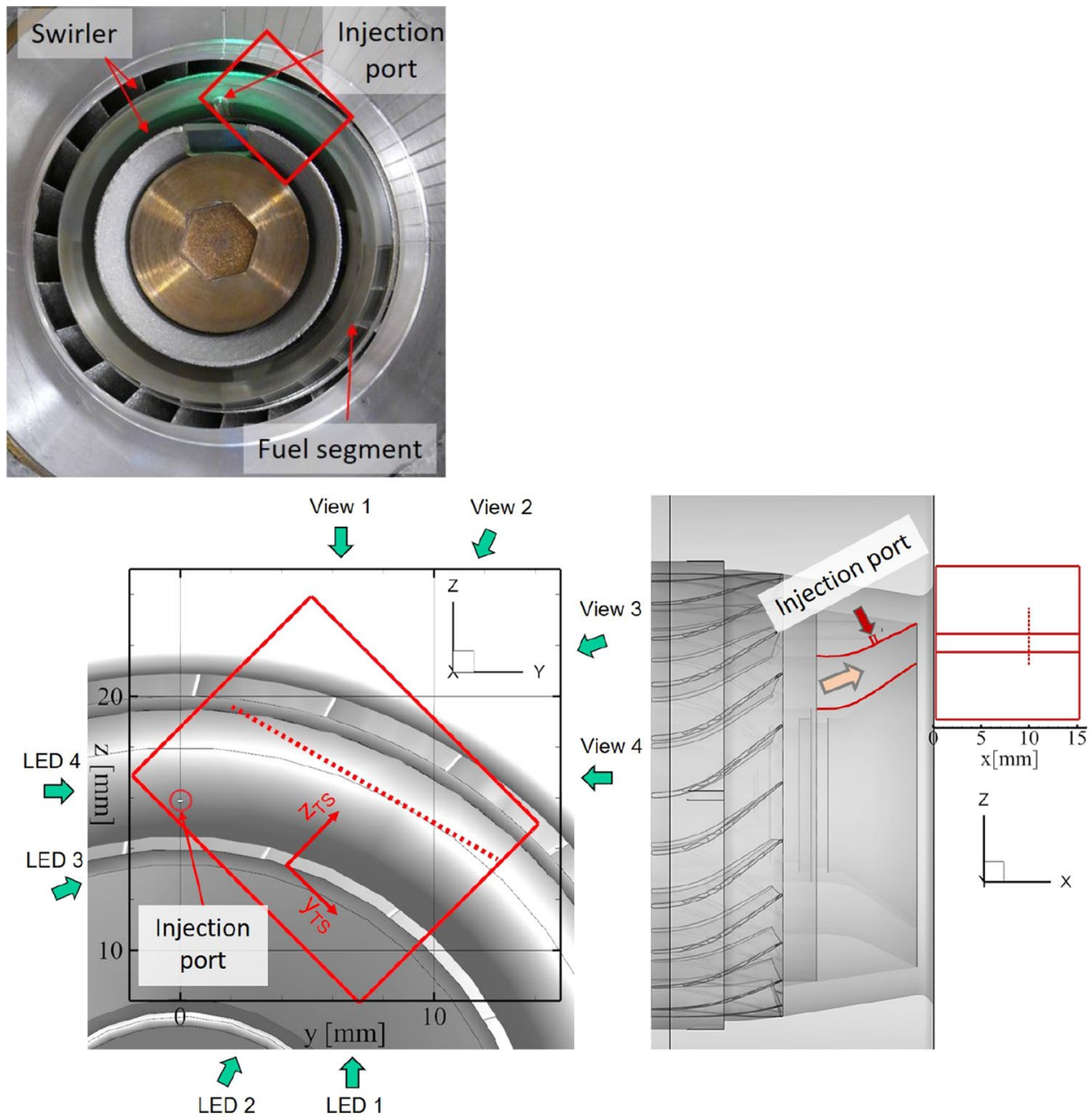

with an aspheric condenser lens of $f=30 \mathrm{~mm}$. In order to achieve short bright light pulses, the LEDs are operated in overdriven pulsed mode (Willert et al. 2010) with drive currents of $I_{\mathrm{f}}=270 \mathrm{~A}$ and a duration of $400 \mathrm{~ns}$. With the camera lenses stopped down to $f_{\#}=22$, brightfield intensity levels of $5 \%$ of the camera dynamic range (16 bit) are achieved. An effective FWHM pulse duration of $t_{p}=540 \mathrm{~ns}$ was determined using a fast response photo diode (Thorlabs, DET10A) and is due to the finite rise and fall times of the LED's luminosity (cf. Fig. 6). The temporal lag of the light emitted by LED in response to a nearly rectangular current drive pulse was already observed in previous applications (cf. Willert et al. 2010, 2012; Nasibov et al. 2014; Giskes et al. 2016) and mostly can be attributed to the inductance and parasitic capacitance of the utilized driver circuitry which is not further described herein. Due to the high current, the LED's emission spectrum also shifts from $\lambda_{\max }=533 \mathrm{~nm}$ to $\lambda_{\max }=514 \mathrm{~nm}$ while maintaining a spectral bandwidth of $38 \mathrm{~nm}$ (FWHM). The spectrum was measured with a spectrometer of $0.34 \mathrm{~nm}$ resolution (Ocean Optics USB2000+).

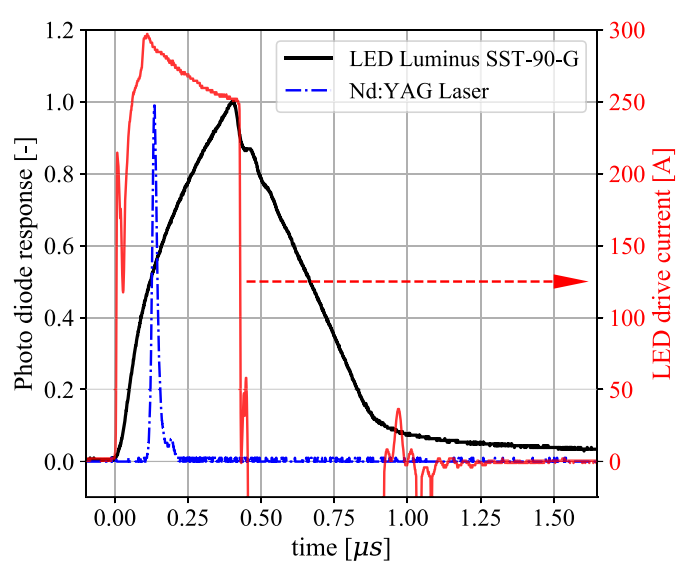

Fig. 6 Response of green Luminus SST-90 LED to a single current pulse of $400 \mathrm{~ns}$ duration as monitored by a photodiode. The dashed trace shows the photodiode response to a light pulse of an Nd:YAG laser of $8 \mathrm{~ns}$ duration 
Table 2 Imaging parameters for tomographic shadowgraph imaging; viewing angles are provided with respect to the plane $x_{T S}-z_{T S}$ (Fig. 5) which corresponds to $\varphi=0^{\circ}$

\begin{tabular}{llllll}
\hline $\begin{array}{l}\text { View } \\
\text { No. }\end{array}$ & $\approx \varphi$ & $\begin{array}{l}\text { lens } f_{\#} \\
{[-]}\end{array}$ & $\begin{array}{l}f \\
{[\mathrm{~mm}]}\end{array}$ & $\begin{array}{l}\approx M \\
{[\mu \mathrm{m} / \mathrm{pixel}]}\end{array}$ & $\begin{array}{l}d_{\text {Airy }} \\
{[\mu \mathrm{m}]}\end{array}$ \\
\hline 1 & $-45^{\circ}$ & 22 & 105 & 7.6 & 51 \\
2 & $-20^{\circ}$ & 22 & 200 & 6.8 & 54 \\
3 & $25^{\circ}$ & 22 & 200 & 6.9 & 53 \\
4 & $45^{\circ}$ & 22 & 105 & 7.1 & 53 \\
\hline
\end{tabular}

In order to capture the droplet displacement between two image exposures, the LED pulse separation is set to $\Delta t=1.7 \mu \mathrm{s}$. At the given magnification this limited droplet image displacements to 20 pixel for velocities in the $80 \mathrm{~m} / \mathrm{s}$ range.

The camera imaging parameters are summarized in Table 2. Image magnifications range from $M=0.85-0.95$ corresponding to $6.8-7.6 \mu \mathrm{m} / \mathrm{pixel}$ in object space. The two outer cameras no. 1 and no. 4 use lenses of $f=105 \mathrm{~mm}$ focal length and $35 \mathrm{~mm}$ extension rings to maintain magnifications near unity. To achieve a longer working distance, the two inner cameras are equipped with $f=200 \mathrm{~mm}$ lenses in order to accommodate the additional compensator plates in the optical path while maintaining a similar magnification compared to view no. 1 and no. 4.

With the aid of Scheimpflug tilt mounts, the focal planes of the cameras are aligned with the calibration target positioned in $x_{T S}-y_{T S}$-planes at the center of the imaged volume (cf. Fig. 5). The calibration plate is aligned parallel to the $x_{T S}-y_{T S}$ - plane and translated along the $z_{T S}$. Each calibration set consists of seven $z_{T S}$ positions spaced at $1.5 \mathrm{~mm}$. Point correspondences of the calibration markers are used to obtain parameters of a higher order camera model which applies ratios of second order polynomials to compensate for lens distortions (for details see Klinner 2017).

\subsection{Compensation of optical aberrations introduced by thick pressure windows}

While the viewing directions of the outer cameras no. 1 and no. 4 are perpendicular to the facility windows, cameras no. 2 and no. 3 have to be tilted with respect to these windows. This results in optical aberrations that renders droplet images of both with elliptical distortions. Analytical expressions for such aberrations imparted by a tilted planeparallel plate to a converging pencil of rays are given by Braat (1997). Compared to spherical and coma aberrations, the oblique view through the thick windows at angles of $\beta=20-25^{\circ}$ introduces by far strongest optical aberration in the form of astigmatism. The wavefront aberration coefficient of this astigmatism is proportional to $l \mathrm{NA}^{2}$ with $l$ being the window thickness and NA being the numerical aperture (see Braat 1997). The astigmatism of the tilted window causes orthogonal lines in a object plane to focus sharply at different distances in the image space. This aberration effect is illustrated in Fig. 7, where a ray tracing was performed in air through a tilted quartz plate of 42 -mm-thickness $\left(\beta=25^{\circ}, n=1.46\right)$ and through a biconvex lens $(f=2 l$, $M=1, \mathrm{NA}=0.1)$. Here the pencil of rays in the $x^{\prime}-z^{\prime}$ plane focus at a different position compared to the pencil of rays in the $y^{\prime}-z^{\prime}$ plane with the foci separated by $\Delta s^{\prime}$. Given the imaging parameter of view no. 2 (see Table 2), a total window thickness of $l=42 \mathrm{~mm}$ and a tilt angle of $\beta=25^{\circ}$, the tracing of the extreme rays in the numerical aperture of the lens would result in a back-focal displacement of $1.4 \%$ of the focal length or $\Delta s^{\prime}=2.8 \mathrm{~mm}$. The corresponding lateral defocusing would be in the order of $2 \mathrm{NA} \Delta s^{\prime}=65 \mu \mathrm{m}$ or about 10 pixels.

The astigmatic distortion from a tilted glass plate in the image plane is typically corrected using cylindrical lenses, whereby curvature radius and refractive index are determined from the expected astigmatic focus difference (Zheng et al. 2021). Inherently, in a convergent beam path the astigmatism correction by a cylindrical lens has to be adjusted according to specific inclination angles (i.e., a specific working distances for one-to-one imaging) and does not compensate for astigmatism along the entire depth-of-field.

In order to achieve adequate compensation of the astigmatism over the entire depth of the measurement volume of $10 \mathrm{~mm}$, plane-parallel glass blocks with the same optical length as the pressure and liner windows were used here instead of cylindrical lenses, which produce similar astigmatism as pressure and liner windows when tilted through the same angle. Once rotated into a perpendicular position with respect to the plane of the facility window image distortions can be nearly eliminated. The working principle of the compensator is illustrated by the ray tracing in Fig. 8. Here, the beam path of two extreme rays originating from the same

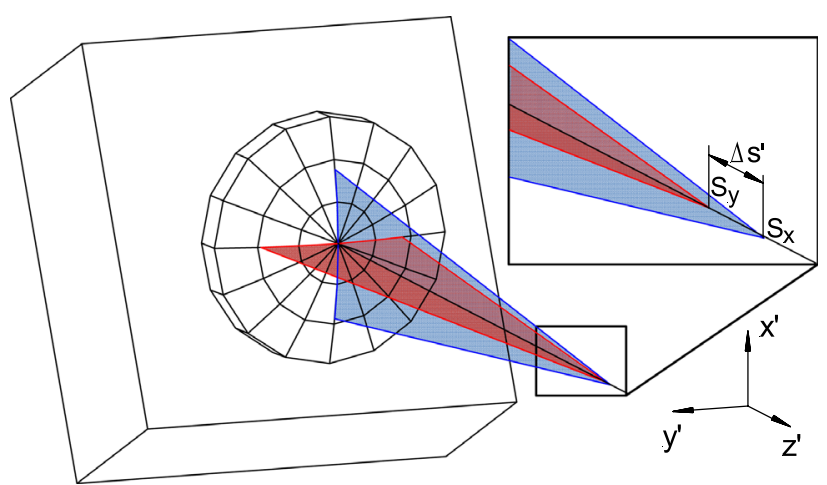

Fig. 7 Astigmatism in the image plane due to observation through a thick tilted glass block representing a pressure window 


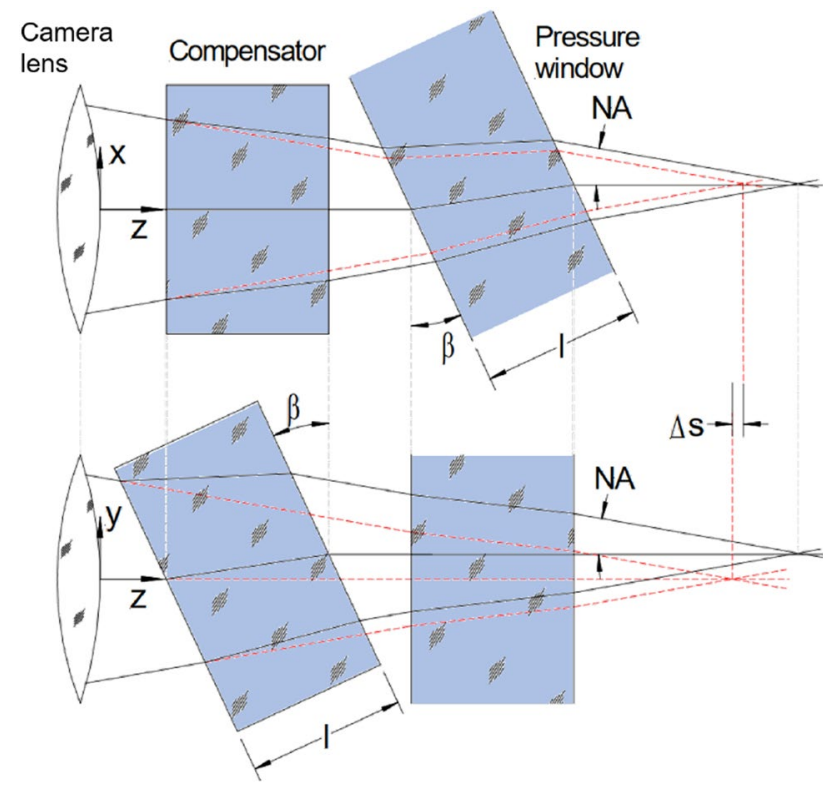

Fig. 8 Working principle of astigmatism compensation in the object space (rays originate from the same point in image space); dashed red line: beam path without compensator; black lines: beam path with compensation

point in image space are shown in perpendicular propagation planes and with and without compensation. The tilt angle $\beta$ of the plate is equal to the tilt angle of the windows but while the camera tilt axis is parallel with the $y$-axis the compensator tilt axis is parallel with the $x$-axis. This leads to cancelation of the focal displacement $\Delta s$.

The performance of the compensator block was verified qualitatively with spray shadowgraphs of a full cone water spray illuminated with pulsed LED illumination. Astigmatic distortions are clearly visible when the spray is imaged through a 40-mm-thick glass window with a tilt of $30^{\circ}$ (Fig. 9a). The majority of the droplets are either imaged as vertical or horizontal ellipses. With the introduction of the compensator glass block as described above, additional elliptical droplet image distortions are introduced with a different orientation such that the droplet images become circular throughout the imaged domain of $10-\mathrm{mm}$-depth (Fig. 9b).

\section{Data processing}

\subsection{Image pre-processing}

Prior to 3-d reconstruction the droplet images acquired with the tomographic imaging system are subjected to image enhancement. After dark image subtraction, the images are median filtered using a $3 \times 3$ kernel to reduce pixel artifacts of the sCMOS camera sensor. Flat-field correction and normalization of the images are performed by division with a bright field image without spray. Finally, the image intensities are inverted, and a constant offset of $2 \sigma$ is subtracted to clip intensities close to pixel noise (pre-processing method $\mathbf{A}$ in Fig. 10b middle). The remaining unstructured background between droplet shadows is believed to originate from small vaporized kerosene droplets with sizes below the detection limit or from droplets which are well out-offocus. Unstructured background intensity around droplet images could be partially removed by subtracting the local minimum in a $20 \times 20$ pixel kernel followed by clipping of a constant threshold (pre-processing method $\mathbf{B}$ in Fig. 10b bottom). Pre-processing method A without sliding minimum subtraction and thresholding is applied prior to reconstructions that are used to gain information about the placement of liquid phase in the spray (spatial intensity distribution). Pre-processing method $\mathbf{B}$ is optimized to enhance gradients near the droplet shadow border and to improve the correlation signal for droplet velocity estimation.

\subsection{3-d reconstruction of the spray field}

The observed intensities from each voxel are reconstructed according to the line-of-sight intersection with each image plane. These positions are calculated using mapping
Fig. 9 a Astigmatic distortions of a shadowgraph of a full cone water spray when observed through a glass window of 30-mm-thickness under an viewing angle of $30^{\circ}$ $\left(f_{\#} 16, M=1\right)$. b Shadowgraph recorded under the same conditions but with an additional compensator block

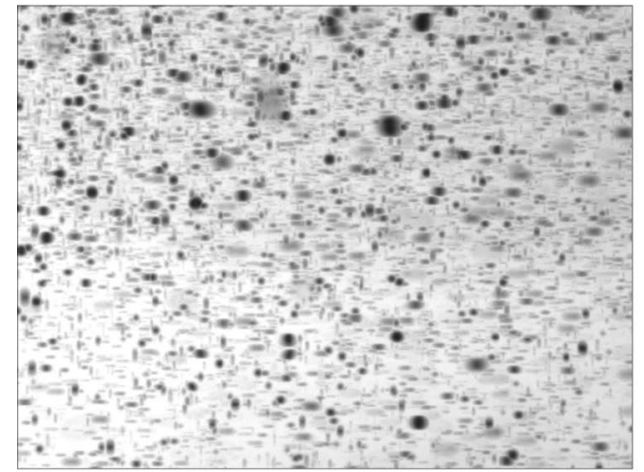

(a)

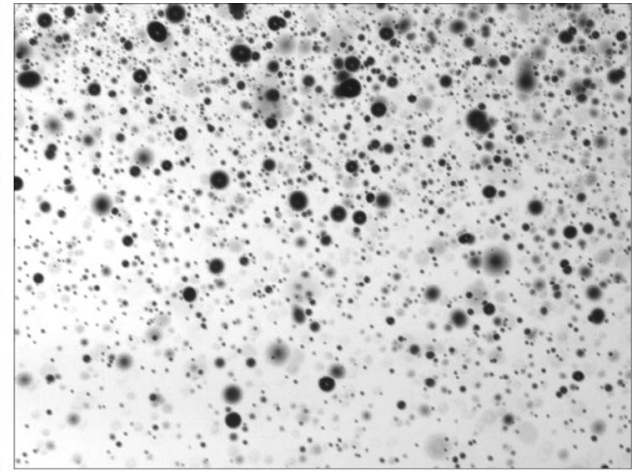

(b) 


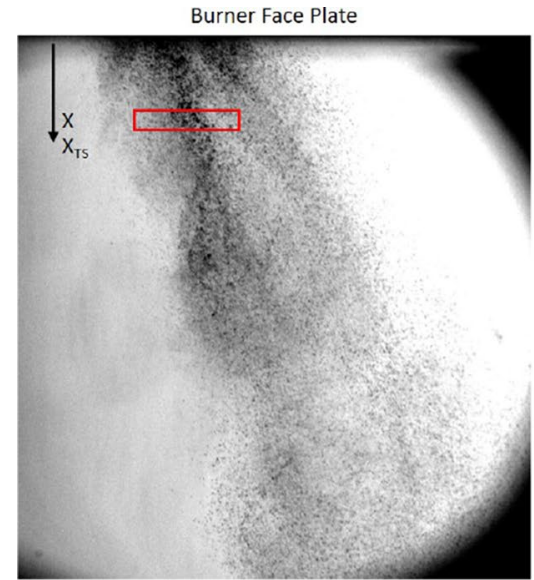

(a)

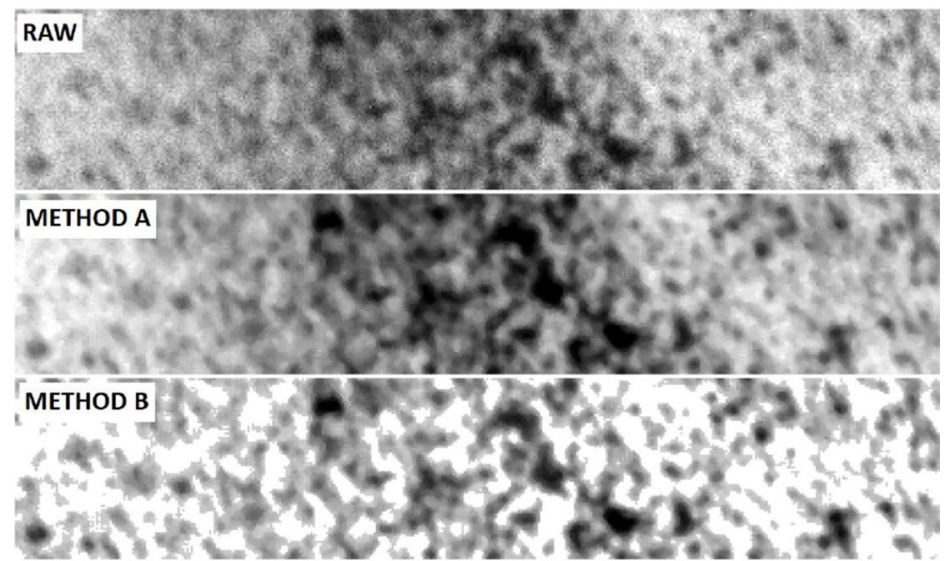

(b)
Fig. 10 a Sample spray image obtained from view no. 1 at $p_{a}=4$ bar, $T_{a}=440 \mathrm{~K}, q=8$, We $=360$. b Magnified region of $410 \times 80$ pixels $(\approx 3.11 \times 0.61 \mathrm{~mm})$ of highlighted area in (a) (red

functions of ratios of second order polynomials Klinner (2017) which are obtained from camera calibration, and the observed intensities from each view are combined by a 2-d maximum entropy technique (MENT) as described by Bilsky et al. (2013). The basic idea behind MENT is that the intensities are summed along the 2-d epipolar line, which is the projection of the line of sight in each corresponding images. The main advantage is that the computation and storage of voxel intensities are not required during the iterative refinement process. Details regarding the applied implementation can be found in Klinner (2017). Here it should be noted that the use of MENT for 3-d droplet field reconstruction was more based on availability rather than performance. Other reconstruction methods such as the multiplicative algebraic reconstruction technique MART (Elsinga et al. 2006) and variants thereof are likely to produce better results, in particular, with respect to the suppression of reconstruction ambiguities (i.e., "ghost droplets"). On the other hand, the performance of the more advanced methods often relies on having nearly uniform "particle" image sizes and intensities throughout the images which is not the case for the present polydisperse droplet image data. In this regard, the MENT technique may be more robust.

The resolution assessments provided in the following section provide a theoretical upper bound (i.e. what is possible) neglecting the influence of reconstruction ambiguities whose efficient suppression is subject to future work. The resolution assessment outlined in the preceding section shows that a voxel size of $12 \mu \mathrm{m}$ represents a sufficient spatial sampling during reconstruction. For a volumetric domain of $16 \times 13 \times 10 \mathrm{~mm}^{3}$, this corresponds to a total size of $1333 \times 1083 \times 833$ voxels. For image sampling, sub-pixel box). Top: raw image; Middle: after contrast enhancement (method A); Bottom: after local minimum subtraction $(20 \times 20$ neighborhood $)$ and thresholding (method $\mathbf{B}$ )

intensities are bi-linearly interpolated between the 4-connected pixels.

\section{Resolution assessments}

\subsection{Depth of field and smallest visible drop size}

Tomographic shadowgraph imaging relies on shadow images or shadowgraphs of a spray field using polychromatic inline illumination with pulsed LED light. The term 'shadow image' does not fully address the involved processes because the absorption coefficient of kerosene (Jet-A1) for visible light is rather low $\left(5.0 \times 10^{-7}\right.$ at $20^{\circ} \mathrm{C}$ and $1.7 \times 10^{-6}$ at $280^{\circ} \mathrm{C}$; Rachner 1998 ). Most of the photons interacting with the droplet are deflected by reflection, refraction and diffraction such that images of small droplets appear as dark spots on a bright background because the majority of the deflected light is not captured by the imaging lens (see Fig. 11).

This raises the question on how the lens aperture, optical resolution and the further image processing affects the shadow image contrast and thus droplet visibility. PDA measurements $10 \mathrm{~mm}$ downstream of the burner face plate revealed kerosene droplet diameter ranging from 5-80 $\mu \mathrm{m}$ for base conditions at OP no. 1 (cf. Table 1). However, it is not obvious which part of this size fraction can be captured by tomographic shadowgraph imaging.

In the following, the visibility of spherical droplet shadows as a function of droplet size and volume position $z_{T S}$ is estimated for the previously described multiple view setup. The underlying spray imaging model is described by Blaisot and Yon (2005) who established the model to enable measurements of droplet sizes within diesel sprays using 


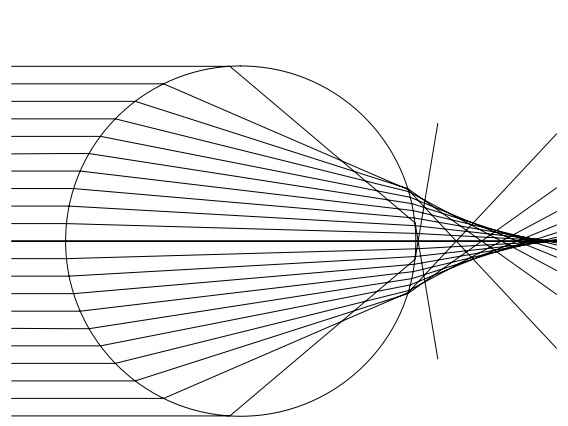

(a)

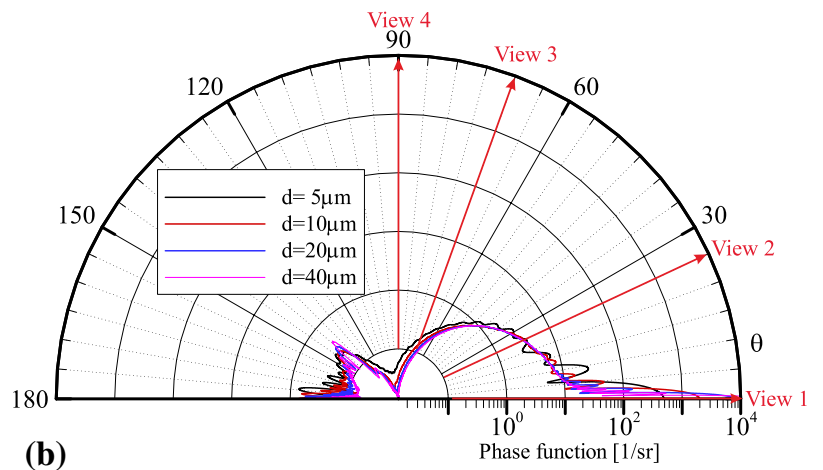

(b)

Fig. 11 Light refraction inside a sphere (a) and Mie scattering of non-polarized polychromatic LED light $(\lambda=460-600 \mathrm{~nm})$ upon spherical kerosene droplets in air (b), Indicated viewing directions with respect to LED no. 1

shadowgraphs obtained from a single camera and noncoherent backlight illumination.

For the modeling of droplet imaging according to Blaisot and Yon, a contrast estimation is necessary, which can only be done here by neglecting the multiple scattering in a dense spray. Furthermore, the direction dependence of the illumination also plays a role in image-based droplet sizing which requires a re-calibration of Blaisots model using images of defined micro-discs of various sizes (Fdida and Blaisot 2010). In the following estimation of the droplet contrast and upper bound of droplet visibility, variations of the incidence angle of the LED light are neglected which are effectively $\pm 50 \mathrm{mrad}$.

In the imaging model of Blaisot and Yon, the radial intensity distribution of a droplet shadow is modeled by the convolution of a slightly transmitting disc and a point spread function (PSF) with the latter describing the resolving capability of the imaging system. In the imaging model, the PSF is considered as a Gaussian and can be calibrated as a function of $z_{T S}$ :

$s\left(r, z_{T S}\right)=s_{0} \exp \left(-\frac{2 r^{2}}{\chi^{2}\left(z_{T S}\right)}\right)$,

where $s_{0}$ represents a normalization factor, $r$ is the radial droplet image coordinate, and $\chi$ corresponds to the width at about $60 \%$ of the full height $(2 \sigma)$.

The convolution of the slightly transmitting disc and the Gaussian PSF leads to:

$i_{n}(\tilde{r})=1-2(1-\tau) \exp \left(-\tilde{r}^{2}\right) \int_{0}^{\tilde{a}} \exp \left(-\rho^{2}\right) I_{0}(2 \tilde{r} \rho) d \rho$,

where $\tilde{r}$ is the non-dimensional radial image coordinate $(\tilde{r}=\sqrt{2} r / \chi)$, and $\tilde{a}$ is the non-dimensional droplet image radius $(\tilde{a}=\sqrt{2} a / \chi)$ both with regard to the half width of the PSF; $I_{0}$ is the Bessel function of the first kind and order zero. The contrast coefficient $\tau$ refers to the disc transmission or Mie scattering within the bounds of the lens aperture. If not calibrated, the estimation of the smallest visible droplets requires assumptions concerning the contrast coefficient $\tau$ which are obtained from Mie simulations using MiePlot 4.5 (Laven 2003).

Figure 12 a shows the simulated forward-scattered intensity as a function of scattering angle for polychromatic LED light $(\lambda=460-600 \mathrm{~nm})$ of random polarization upon spherical kerosene droplets at temperatures of $T_{k}=450 \mathrm{~K}$ and air temperatures of $T_{a}=473 \mathrm{~K}$. The scattered intensity is expressed in terms of the so-called phase function, which, when integrated over the full solid angle, yields unity. While scattering by small droplets of $d=10 \mu \mathrm{m}$ does not show a strong angle-dependency, larger droplets of $d=40 \mu \mathrm{m}$ exhibit pronounced forward-scattering with an on-axis intensity fifteen times higher and an additional intensity minimum at a scattering angle of $15.5 \mathrm{mrad}$. If absorption is neglected, integration of the phase function over the solid angle of the camera aperture yields the droplet transmission versus lens aperture angle which is plotted in Fig. 12b. Lenses were stopped down to $f_{\#}=22$ which gives an aperture angle of $11 \mathrm{mrad}$ at a magnification of $M=1$. For this numerical aperture, droplets of diameter $d=10 \mu \mathrm{m}$ or $d=40 \mu \mathrm{m}$ would, respectively, have contrast coefficients of 0.05 and 0.40 . Within the finite lens aperture, light scattering by droplets therefore has a strong droplet size dependency.

In the present multi-LED inline setup, each camera may additionally receive side-scattered light from other LEDs. However, these intensities are significantly smaller compared to the forward-scattered light. In Fig. 11b, the scattering characteristics for LED no. 1 are shown with arrows indicating the respective camera viewing directions. For droplets around $5 \mu \mathrm{m}$ and a small scattering angle of $25^{\circ}$, view no. 2 receives less than $1.5 \%$ from LED no. 1 and LED no. 3 in relation to forward scattered light which originates from LED no. 2 (cf. Fig. 5). The side-scattering further reduces as the droplet size increases, such that the attenuation of the contrast coefficient by side-scattered LED light can be neglected in the following. 

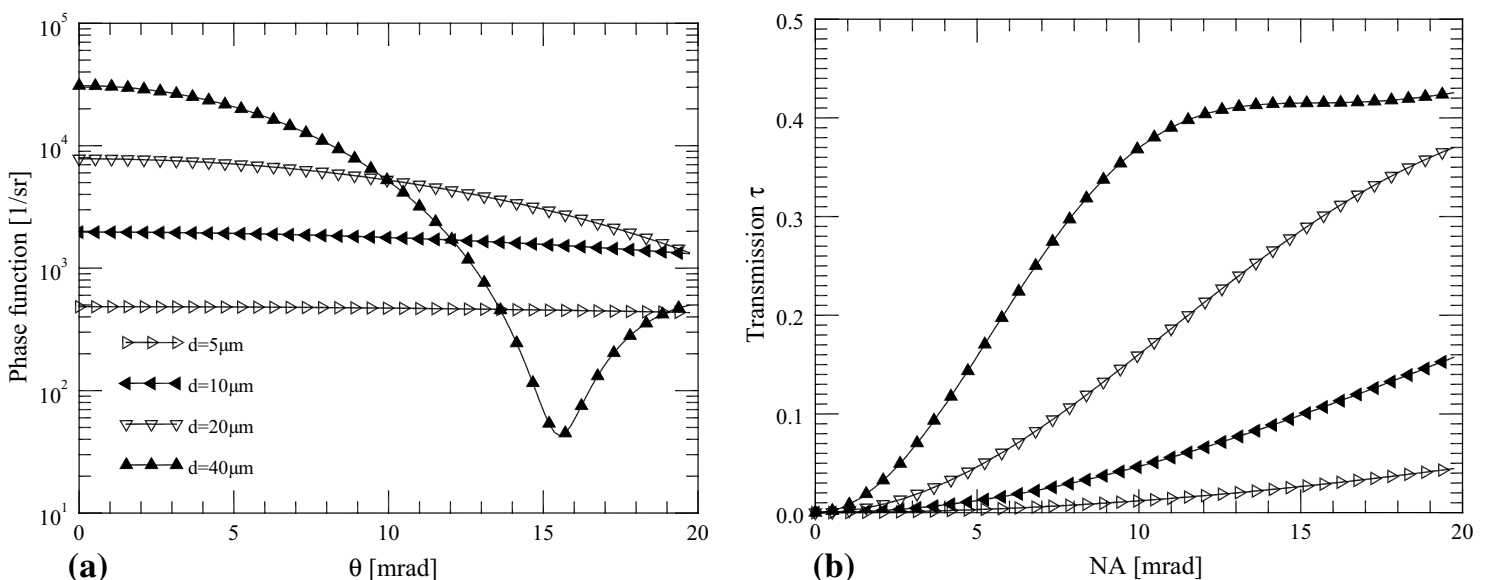

Fig. 12 Forward-scattered intensity versus scattering angle of non-polarized and polychromatic light $(\lambda=460-600 \mathrm{~nm})$ upon spherical Jet-A1 droplets in air at $T_{k}=450 \mathrm{~K}, T_{a}=473 \mathrm{~K}$ (a) and droplet transmission derived from the phase function integrated over the lens aperture (b)
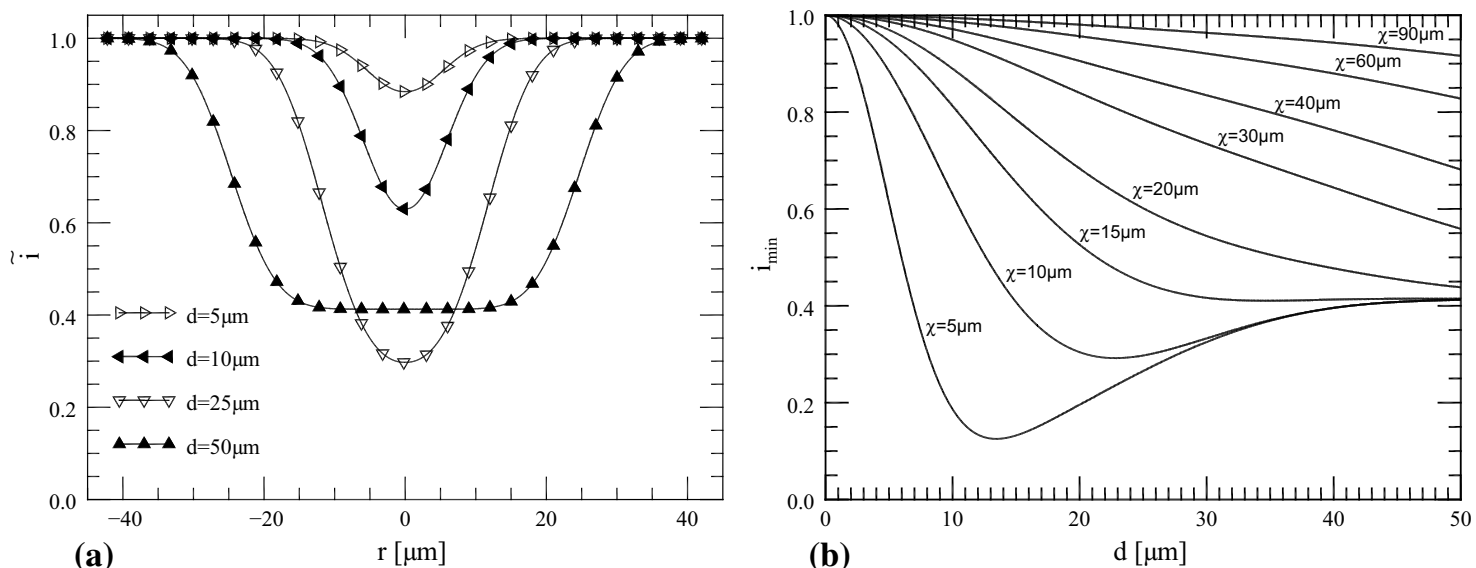

Fig. 13 a Simulated shadow image profiles of kerosene droplets of different sizes at a PSF half-width of $\chi=10 \mu \mathrm{m}, M=1, f_{\#}=22$. b Minimum intensity of droplet image profiles versus droplet size at different PSF half-widths at $M=1, f_{\#}=22$

Based on estimates of the contrast coefficient, the intensity profile of spherical kerosene droplets of different diameters can be simulated. For example, Fig. 13a shows image profiles for a width of a Gaussian point spread function of $\chi=10 \mu \mathrm{m}$ and a magnification of $M=1$. Figure 13b shows the change of the intensity minimum of the image profile with droplet size for different PSF widths.

The shape and contrast of the droplet images can also be affected by the finite duration of the illumination pulse (see Fig. 6). With increasing droplet velocity, the images will exhibit streaking such that the recorded droplet images will no longer be circular.

Another unknown is the shape and width of the point spread function (PSF). Due to the use of Scheimpflug mounts, focal planes of all views are almost parallel with respect to the calibration plate. Astigmatic aberrations are minimized by compensator blocks. Hence, the PSF at each depth position should have almost a circular shape with its half-width being equal to half-width of the line spread function (LSF) (Rao and Jain 1967). In order to assess the LSF, intensity profiles across sharp edges (cf. Jones 1958) are extracted from chessboard calibration images recorded at 13 positions along $z_{T S}$ (Fig. 5) with $0.75 \mathrm{~mm}$ spacing within the spray chamber.

The chessboard pattern was positioned inside the spray chamber after each test and images were processed in the same way as the spray images including flat field correction, back-projection and image interpolation with the exception of the local minimum subtraction which suppresses unstructured background in the spray images (processing B). Resolution estimates based on these chessboard images therefore take into account disturbances caused by the optical setup (i.e., thick facility windows and lenses) and by most of the image processing, but exclude additional contrast reductions 
due to multiple scattering, droplet image streaking or density gradients. The latter are assumed to have a minor impact on image quality due to isothermal conditions as explained further below.

After pre-processing, the chessboard images are flat-field corrected and back-projected into the volume to enable an estimation of the width of the overall point spread function in voxel-space which includes possible smoothing effects during reconstruction due to the finite voxel size and image interpolation. Figure 14a shows the intensity profile across chessboard edges (edge spread function) obtained from back projected calibration images of camera no. 1 . The line spread function is obtained by numerical differentiation of the edge spread function (Claxton and Staunton 2008). The halfwidth of the line spread function is equal to the width of the PSF in $x$-direction and is obtained by matching the derived intensity profile with a Gaussian fit (Rao and Jain 1967).

Figure 14b shows an approximately Gaussian shape of the LSF in focus corresponding to $\chi=3.75 \operatorname{voxel}(30 \mu \mathrm{m})$

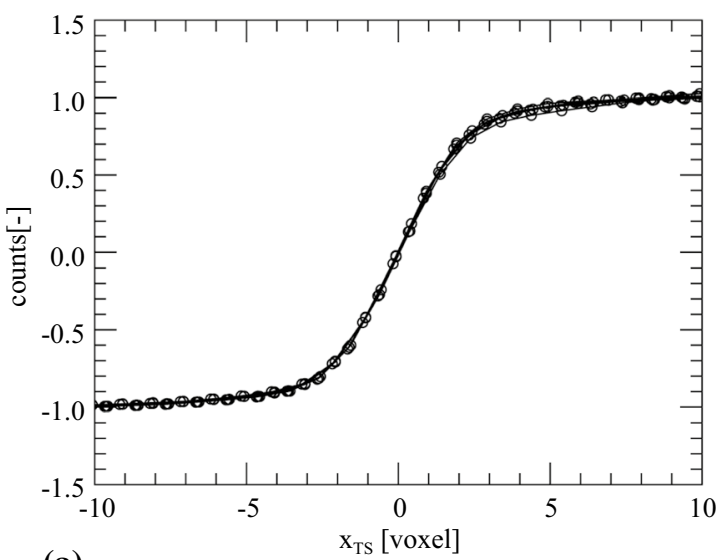

(a)

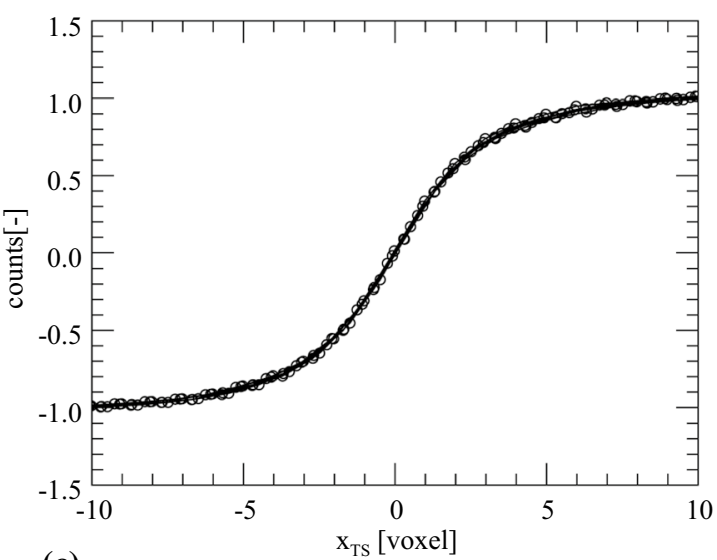

(c)

Fig. 14 Quantification of edge response of the system along the central three chessboard periods along $x_{T S}$ (1/3 of volume width) at a voxel size of $8 \mu \mathrm{m}$ (approx. 1 voxel/pixel) obtained from chessboard images of view no. 1; edge spread (a,c) and line spread function plus with slight additional deviations from the Gaussian at the tails. As expected, in (d) the width of the LSF increases for regions that are out of focus near the volume edge at $z_{T S}=-4.5 \mathrm{~mm}$ to $\chi=5.0 \operatorname{voxel}(40 \mu \mathrm{m})$. This is accompanied by a stronger deviation from the Gaussian with pronounced tails indicating a more Lorentzian shape and a nonideal LSF. In addition to pure diffraction at the lens aperture at f-number 22 (i.e., diffraction limit), geometric aberrations of the lens and windows, as well as internal reflections from the thick glass blocks, are more significant in out-of-focus regions and likely create a halo that leads to a redistribution of intensity from the brighter side of the edge to the darker side, resulting in the larger deviation from the Gaussian shape after differentiation. It is assumed that this contrast reduction in the form of a halo also occurs when imaging the spray outside the lens focus, but this was not taken into account in the parameterizations of the PSF in order to be able to estimate the resolution with the simplified Gaussian imaging model by Blaisot and Yon.

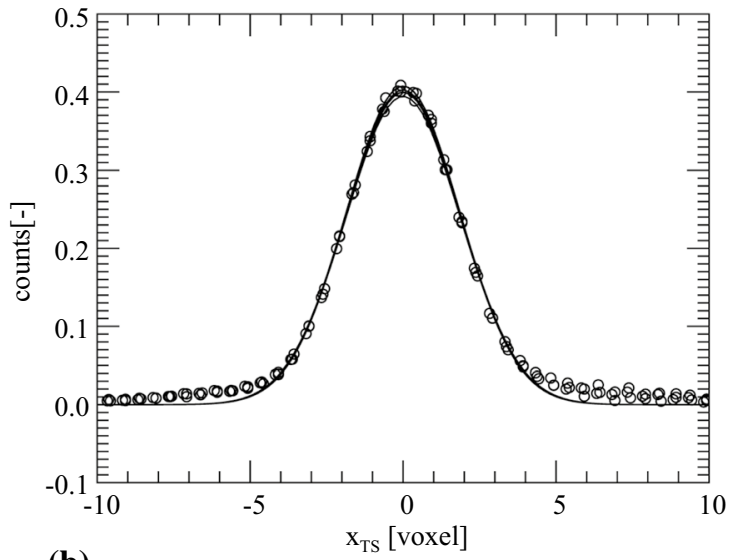

(b)

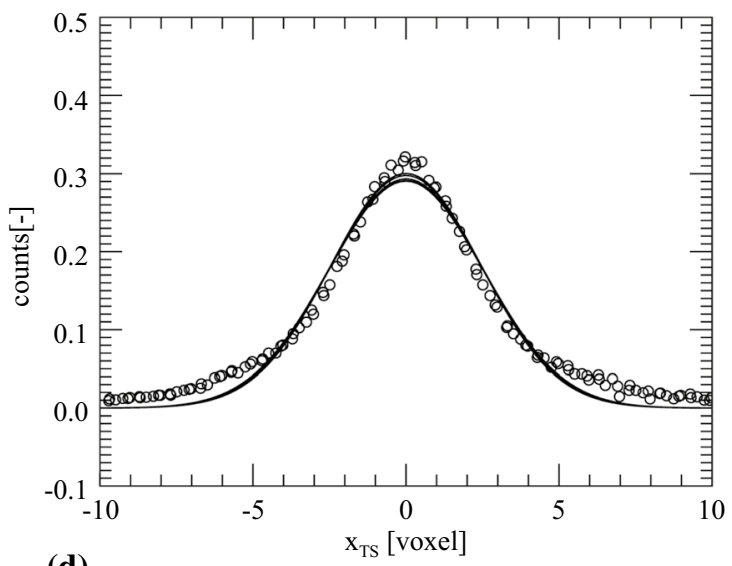

(d)

Gaussian fit (b,d) in focus at $z_{T S}=0 \mathrm{~mm}(\mathrm{a}, \mathrm{b})$ and near the volume edge at $z_{T S}=-4.5 \mathrm{~mm}(\mathbf{c}, \mathbf{d})$. The line spread function is obtained by differentiating the edge spread function 

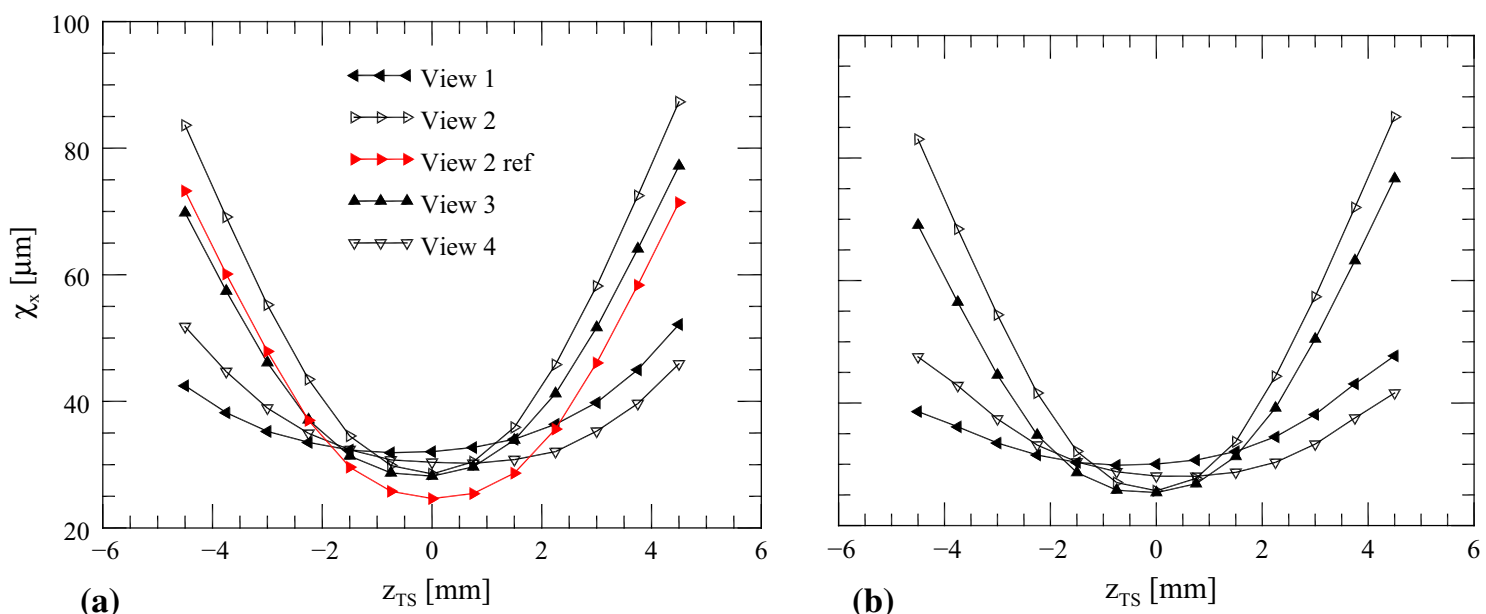

(b)

Fig. 15 Mean half-width of the line spread function along the $z_{T S}$ axis (see Fig. 5); a voxel size of $12 \mu$ m (approx. 1.5 voxel/pixel); b voxel size of $8 \mu \mathrm{m}$ (approx. 1 voxel/pixel); The red trace indicates the measured line spread function without window and compensator block

Figure 15 a shows the mean width of the point spread function obtained from edge response measurements at a sampling of $83 \mathrm{voxel} / \mathrm{mm}$ (voxel size $12 \mu \mathrm{m}$, approx. $1.5 \mathrm{voxel} / \mathrm{pixel}$ ) along the $x_{T S}$ axis (coordinate system as shown in Fig. 5). The image sharpness of the two inner views no. 2 and no. 3 decreases stronger toward the volume edges as compared to view no. 1 and no. 4 . The reference curve (red line) is measured for view no. 2 without windows and without compensator block. The reference exhibits nearly the same shape, indicating that the stronger image blur toward the volume edges is specific for the particular depth-of-field of the lens that has been used for view no. 2 and no. 3 (Nikon Micro Nikkor $f=200 \mathrm{~mm}$ ). Only minor improvements can be achieved by increasing the sampling rate to $125 \mathrm{voxel} / \mathrm{mm}$ (voxel size $8 \mu \mathrm{m}$, approx. $1 \mathrm{voxel} / \mathrm{pixel}$ ) because the resolution is limited by the optical arrangement.

On the basis of PSF calibrations and Mie simulations, the minimum intensity of the droplet image profiles can be calculated by the following equation given by Blaisot and Yon (2005)):

$\tilde{i}(0)=\tilde{i}_{\text {min }}=1-(1-\tau)\left(1-\exp \left(-\tilde{a}^{2}\right)\right.$,

whereby the droplet contrast is $C=\left(1+\tilde{i}_{\text {min }}\right) /\left(1-\tilde{i}_{\text {min }}\right)$.

In order to decide at which image contrast droplets would be visible in a shadow image, a threshold of visibility is established which corresponds to the smallest detectable intensity depletion of the spray background illumination which is estimated from intensity fluctuations in 200 images recorded with LED light without spray. Although the intensity of LED background illumination is constant to fractions of a percent, the temperature gradient in the liner windows and in the cooling gap between liner and pressure windows introduce background intensity modulations due to schlieren. In the absence of spray, temporal variations of inline LED illumination of individual pixels at different positions in views no. 1-4 were on the order of $1 \%$ at base conditions (OP1) and increased up to $2 \%$ in OP4. After image normalization, all pixel intensities have a mean of $\tilde{i}=1 \pm \sigma$, and the threshold of visibility was set to a values of $\tilde{i}_{\text {min }}=1-2 \sigma$. Thus, for the present resolution estimates the thresholds of visibility were chosen at $0.960,0.976$, 0.976 and 0.960 . for views no. $1,2,3$ and 4 .

Figure 16 finally shows the estimated smallest visible droplet sizes in each view for a voxel size of $12 \mu \mathrm{m}$ $(83 \mathrm{voxel} / \mathrm{mm}$ ). If one of the views cannot detect a droplet because of low contrast it would not be visible in the reconstructed volume. Therefore, the combined maximum

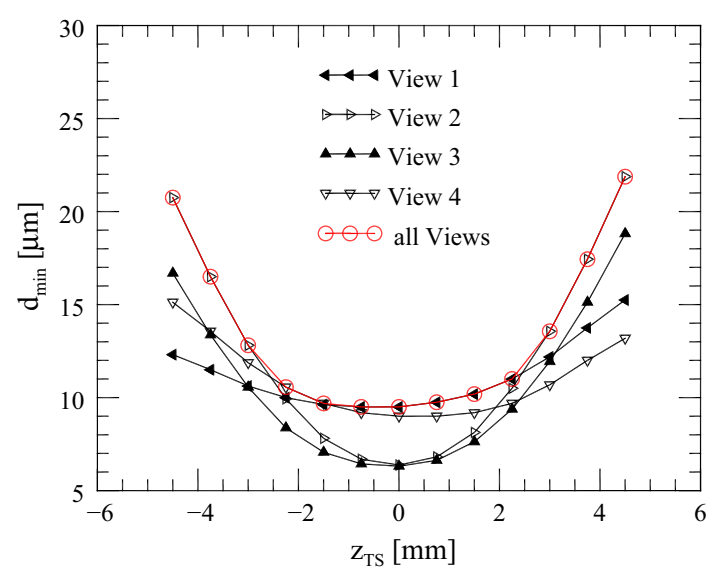

Fig. 16 Upper bound of droplet visibility, i.e., smallest visible droplet diameter, neglecting contrast reduction due to multiple scattering and streaking due to finite LED pulse width at a spatial sampling of $83 \mathrm{voxel} / \mathrm{mm}$ 
of the size limits of all cameras at each calibration plate position gives the limit of the multiple view setup (red line in Fig. 16). For the given volume depth of $10 \mathrm{~mm}$ along $z_{T S}$, the minimum resolvable droplet diameter approaches $d=10 \mu \mathrm{m}$ within the focus and increases up to $d=20 \mu \mathrm{m}$ toward the volume edges.

The droplet visibility is only slightly improved if the spatial sampling rate is increased to $125 \mu \mathrm{m}$ (approx. 1 voxel/pixel) so a sampling of $83 \mathrm{voxel} / \mathrm{mm}$ (voxel size of $12 \mu \mathrm{m}$ ) was chosen to reduce the computational time of volume reconstruction.

\subsection{Estimation of 3-d 3-c droplet velocities and error assessment}

By means of cross-correlation, processing estimates of the droplet displacements are determined from each pair of reconstructed image volume distributions. A multi-grid algorithm employs a resolution pyramid that starts at a coarse grid and stepwise increases resolution while continually updating a predictor field in a similar manner as for planar PIV processing (Soria 1998; Scarano 2002). To increase processing speed, factor $N$ volume down-sampling is applied by summing $N^{3}$ neighboring voxels. At a given resolution level integer-based sample offsetting is applied in a symmetric fashion using the estimate from the previous resolution step. Intermediate validation is based on normalized median filtering as introduced by Westerweel and Scarano (2005). Once the desired final spatial resolution is reached, image or volume deformation based on third-order B-splines (Thévenaz et al. 2000) is applied at least twice to compensate for velocity gradients thereby further improving the match between volumes and corresponding displacement estimates (Astarita and Cardone 2005). The final interrogation volume size of $0.77 \times 0.38 \times 0.38 \mathrm{~mm}^{3}(64 \times 32 \times 32$ voxels $)$ samples the domain with an overlap of $50 \%$ in each spatial direction and results in a corresponding displacement grid with a spacing of $0.38 \times 0.19 \times 0.19 \mathrm{~mm}^{3}(32 \times 16 \times 16$ voxels $)$.

Due to the nature of the data processing (i.e., cross-correlation of sub-volumes), the resulting velocity field can only provide estimates of the group velocity of droplets inside each interrogation box and is not necessarily associated to the velocity of individual droplets.

Furthermore, due to the finite duration of the LED lightpulse (cf. Fig. 6), images of fast moving droplets are elongated in flow direction, resulting in a streak. Due to the finite rise and fall times each of approximately $300 \mathrm{~ns}$, the contrast depletion due to shadow image movement during exposure is not uniform along the streak and has its minimum approximately at a position corresponding to half of the LED pulse width. Streaking was observed in particular for small droplet images further downstream from the burner face plate at operation conditions involving higher temperatures $\left(T_{a}=570 \mathrm{~K}, \mathrm{OP} 2,4\right)$. In this region, the droplet images are elongated approximately by a factor of two. For a 2-d cross-correlation analysis, Ganapathisubramani and Clemens (2006) showed that for the given ratio between pulse-duration and separation $\mathrm{R}=0.540 \mu \mathrm{s} / 1.7 \mu \mathrm{s}=0.32$ streaking can increase the peak detection error approximately by a factor of two for a uniform displacement.

Next, the influence of the specific camera viewing geometry on the error of 3-d displacements is estimated. In the literature, estimates of the influence of camera geometry on the accuracy for tomographic PIV are often based on assessments of the reconstruction quality (quality-factor), where the optimal angle between the outer cameras is estimated as a trade-off between the lowest possible broadening of voxel intensities along $z_{T S}$ and the shortest possible line-ofsight, while the latter is to avoid an increase in reconstruction ambiguities. For a linear camera arrangement as chosen here, Elsinga et al. (2006) found $60^{\circ}$ as the optimal angle between the outer cameras, with the current angle of $90^{\circ}$ still in the optimal range as provided in their paper.

Since the geometry-related accuracy of 3-d displacement estimates cannot be derived directly from the qualify-factor, a different approach is taken here by extending a methodology used for stereoscopic PIV, where the 3-d displacement is decomposed into its projected components in each camera view, for which it is assumed that these projected displacements can be determined with a known accuracy. The proposed approach allows the influence of a particular camera geometry on the accuracy of the 3-d displacements to be estimated independent from other influences such as reconstruction ambiguities. Similarly, the cross-correlation processing used here for each interrogation volume is also based on displacement detections within projections of the voxel space along $x_{T S}, y_{T S}$ and $z_{T S}$, which could equally well be decomposed into projections in each viewing direction.

An expression for the uncertainty of the velocity estimates associated with the specific camera viewing geometry can be derived as follows: Fig. 17 illustrates how each measured velocity component $U_{1}, U_{2}$ depends on Cartesian

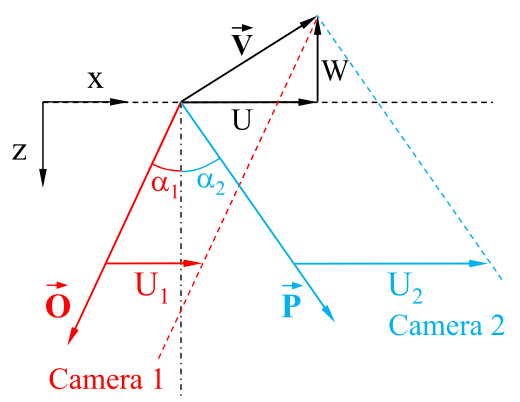

Fig. 17 Stereoscopic measurement of $U$ and $W$ component from two projections (adopted from Raffel et al. 2017) 
particle velocity components in two mapped stereoscopic views, that is:

$U_{1}=-\frac{\Delta X_{I 1}}{M_{1} \Delta t}=U-W \tan \alpha_{1}$

$U_{2}=-\frac{\Delta X_{I 2}}{M_{2} \Delta t}=U-W \tan \alpha_{2}$

Here, $\Delta X_{I 1}$ and $\Delta X_{I 2}$ are the image displacements along $x$ in each camera, $M_{1}$ and $M_{2}$ describe both camera magnifications and $\Delta t$ is the pulse delay. Furthermore, $\alpha_{1}$ and $\alpha_{2}$ describe the angles in the $x-z$ plane between the $z$ axis and the lines of sight through the lens center to the recording plane of a droplet or particle (Raffel et al. 2017). The tangents of $\alpha_{1}$ and $\alpha_{2}$ can also be expressed in terms of components of the observation vectors $\mathbf{O}$ and $\mathbf{P}$ of each interrogation region with $\tan \alpha_{1}=O_{x} / O_{z}$ and $\tan \alpha_{2}=P_{x} / P_{z}$.

In the same manner as provided in Eq. (4), equations can be found for velocity components $U_{i}, V_{i}$ measured by each camera $i$. These equations can be combined to describe a general viewing geometry that is defined through four observation vectors $\mathbf{O}, \mathbf{P}, \mathbf{Q}, \mathbf{R}$ :

$\mathbf{U}_{\text {meas }}=\left[\begin{array}{c}U_{1} \\ V_{1} \\ U_{2} \\ V_{2} \\ U_{3} \\ V_{3} \\ U_{4} \\ V_{4}\end{array}\right]=\left[\begin{array}{lll}1 & 0 & -\frac{O_{x}}{O_{z}} \\ 0 & 1 & -\frac{O_{y}}{O_{z}} \\ 1 & 0 & -\frac{P_{x}}{P_{z}} \\ 0 & 1 & -\frac{P_{y}}{P_{z}} \\ 1 & 0 & -\frac{Q_{x}}{Q_{z}} \\ 0 & 1 & -\frac{Q_{y}}{Q_{z}} \\ 1 & 0 & -\frac{R_{x}}{R_{z}} \\ 0 & 1 & -\frac{R_{y}}{R_{z}}\end{array}\right]\left[\begin{array}{c}U \\ V \\ W\end{array}\right]$.

The system of equation is over-determined, that is, the three unknown velocity components are computed from eight known displacement estimates. Equation Eq. (5) can be solved in a least-squares sense using the pseudo-inverse (c.f. Raffel et al. 2017):

$\mathbf{U}_{\text {meas }}=\mathbf{A ~ V}$

$\Rightarrow \mathbf{V}=\left(\mathbf{A}^{T} \mathbf{A}\right)^{-1} \mathbf{A}^{T} \mathbf{U}_{\text {meas }}=\mathbf{A}^{+} \mathbf{U}_{\text {meas }}$,

Because $\mathbf{V}$ (the actual velocity vector) depends linearly on the measurement vector $\mathbf{U}_{\text {meas }}$ each entry of $\mathbf{A}^{+}$can be considered as first order partial derivative. Assuming that each of the components of $\mathbf{U}_{\text {meas }}$ is uncorrelated that the partial derivatives in $\mathbf{A}^{+}$can be used to estimate the combined velocity uncertainty (Joint Committee for Guides in Metrology (JCGM) 2008):

$$
\begin{aligned}
\sigma_{U} & =\sqrt{\sum_{i=1}^{N}\left[\frac{\partial U}{\partial U_{i}}\right]^{2} \sigma_{U_{i}}^{2}+\sum_{i=1}^{N}\left[\frac{\partial U}{\partial V_{i}}\right]^{2} \sigma_{V_{i}}^{2}} \\
\sigma_{V} & =\sqrt{\sum_{i=1}^{N}\left[\frac{\partial V}{\partial U_{i}}\right]^{2} \sigma_{U_{i}}^{2}+\sum_{i=1}^{N}\left[\frac{\partial V}{\partial V_{i}}\right]^{2} \sigma_{V_{i}}^{2}} .
\end{aligned}
$$

Here, $\sigma_{U_{i}}$ and $\sigma_{V_{i}}$, are the standard deviations of measured velocities in each of $N$ views. These uncertainties are estimated as follows:

$\sigma_{U_{i}}=\sigma_{V_{i}}=\frac{\sigma_{I I}}{M_{i} \Delta t}$

where $\sigma_{I I}$ denotes the uncertainty of displacement detection (cross-correlation noise).

An estimation of $\sigma_{I I}$ is not found to be feasible because the random noise of signal peak detection depends on both the number of spray droplets per interrogation volume and the 3-d size of droplet reconstructions (Westerweel 2000). Both parameters are unknown and depend on the spatial spray distribution, the local size distribution, the number of ghost particles and the local PSF. Based on Eq. (5)Eq. (8), relative uncertainties can nonetheless be provided for the present viewing geometry. Here the random uncertainties on the velocity component along $z_{T S}$ is 1.31 times larger compared to velocity components along $x_{T S}$ and $y_{T S}$ which both are almost equal. In fuel injector coordinates (along $x, y, z$ in cf. Figure 5), this results in uncertainties that are $16 \%$ larger for the $v$ and $w$ components in comparison to the $u$ component which aligns with the burner axis.

Furthermore, the cross-correlation coefficient within each interrogation volume provides a measure of the image contrast of the contributing droplets and ligaments and can be used to localize the boundary of the spray cloud. This allows to exclude regions of low droplet number density and/or low image contrast and is based on rejecting displacement vectors with correlation coefficients below 0.1 .

\section{Results}

As described in Sect. 3.1, high-speed shadowgraph imaging was applied at the glass fuel segment with window insert to survey the initial stages of the spray prior to leaving the swirl passage and entering into the spray chamber (cf. Fig. 3). Figure 18 provides successive shadowgraphs recorded at $54 \mathrm{kfps}$ and shows the break-up of the kerosene jet within the annular duct for increasing Weber numbers. These visualizations confirmed that, at constant $q=8$, the primary jet atomization is induced by the swirling air flow inside the annular channel while ensuring that the fuel jet 
(a) $\mathrm{p}_{a}=4 \mathrm{bar} \mathrm{T}_{2}=440 \mathrm{~K} \quad \mathrm{We}=418$

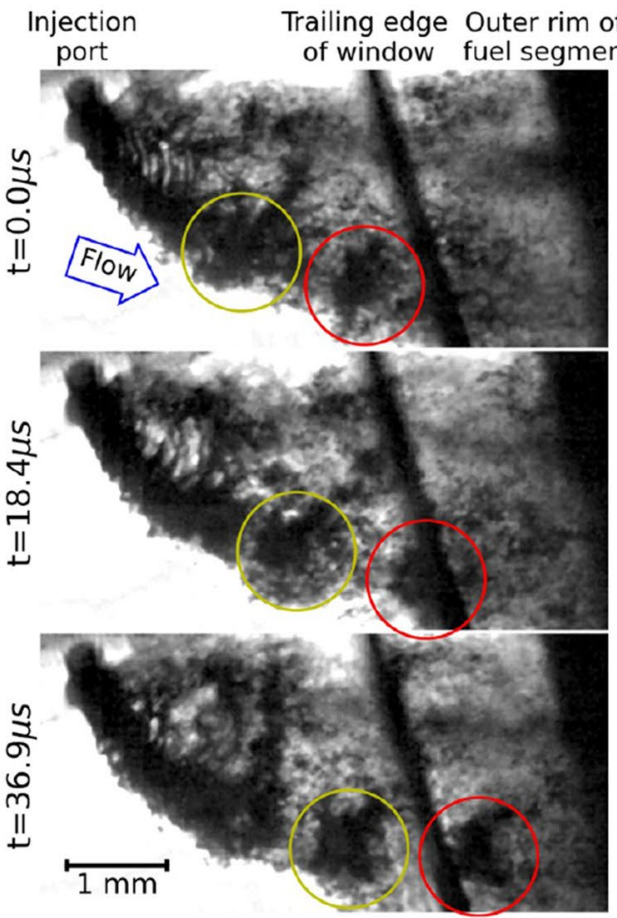

(b) $\mathrm{p}_{a}=4 \mathrm{bar} \mathrm{T}_{a}=570 \mathrm{~K} \quad \mathrm{We}=508$

(c) $\mathrm{p}_{a}=7 \mathrm{bar} \mathrm{T}_{a}=440 \mathrm{~K}$ We $=725$
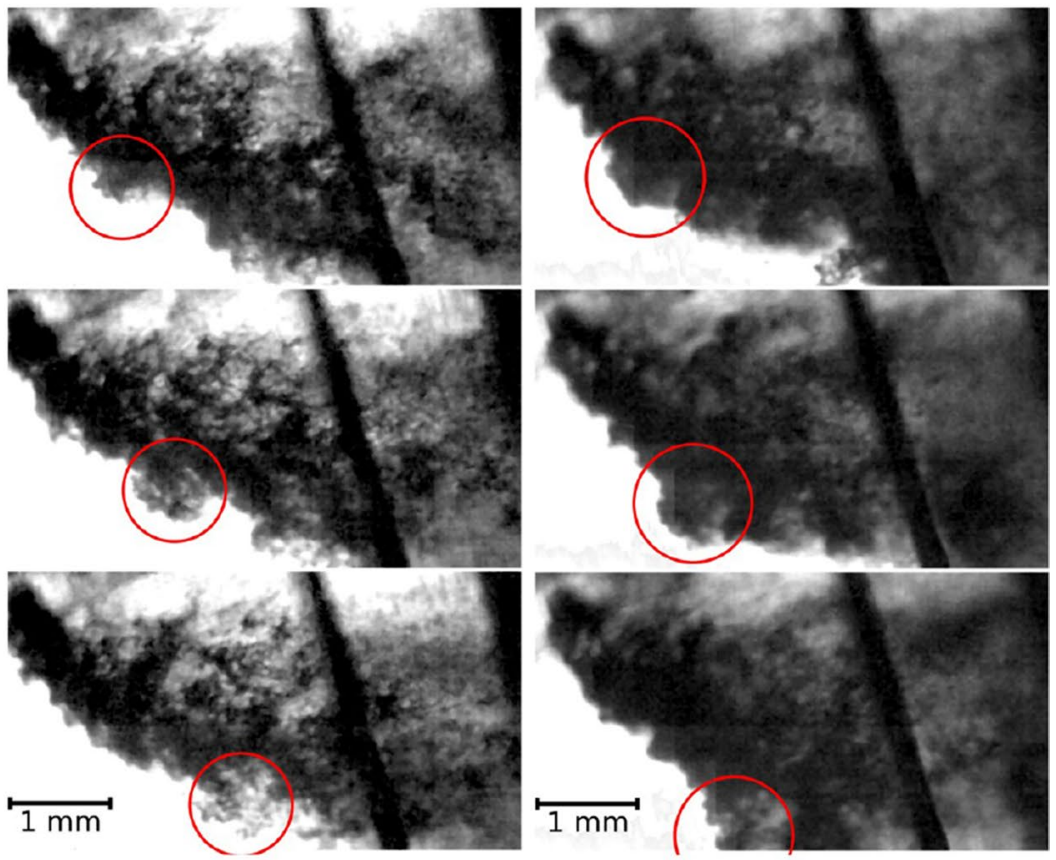

Fig. 18 Successive spray images of the exiting liquid jet $(q=8)$ inside the annular duct recorded with $54 \mathrm{kfps}$; a OP no. 1; circled regions indicate column break-up of the liquid jet; with increasing Weber number shear break-up becomes predominant in b OP no. 2 and $\mathbf{c}$ OP no. 3; The velocity magnitudes of the circled regions are approximately $30-35 \mathrm{~m} / \mathrm{s}$, images have been rotated to match the orientation of the spray injection shown in the following figures. Animated versions of the atomization process are provided in the supplemental material neither impacts the V-shroud on the opposite side nor the fuel segment before exiting the duct.

At the lowest Weber number (see Fig. 18a), column break-up can be observed as indicated within the circled regions which move at convection velocities of approximately $30 \mathrm{~m} / \mathrm{s}$. Additional bag-shaped membranes occur on the flanks of the jet where ligaments and drops are stripped from the liquid surface. This is accompanied by surface waves which develop almost immediately above the nozzle bore. Variations of the position where the liquid column fractures can be observed in the animations provided in the supplemental material.

With increasing Weber number (see Fig. 18b,c), surface waves grow faster and shear break-up becomes the dominant break-up mechanism. The convective velocity of the growing surface waves marked by the red circles increases to magnitudes of approximately $35 \mathrm{~m} / \mathrm{s}$. For liquid jets in cross-flow, the transition Weber number above which surface break-up dominates the column break-up regime can be estimated by $\mathrm{We}_{\mathrm{sb}} \approx 10^{(3.1-\log (q)) / 0.81} \approx 500(\mathrm{Wu}$ et al. 1998; Becker and Hassa 2002). This critical value is between the two conditions visualized in Fig. 18 a and b.
Further downstream and near the outer rim of the fuel segment in Fig. 18 (toward the right edge of the field of view), the spray is at a late state of air-blast atomization as it leaves the annular gap. At this point large fractions of the liquid column are already dispersed into ligaments and droplets. Immediately downstream of the injector face plate, the droplet distribution is inhomogeneous and wavy streaks of larger droplets or lumps of larger droplets appear during the secondary stage of atomization in Fig. 10a.

Figure 19a shows an exemplary instantaneous 3-d spray distribution reconstructed from the multiple view imaging setup using pre-processing method A. Recorded at base conditions ( $p_{A}=4 \mathrm{bar}, T_{A}=440 \mathrm{~K}, q=8$, We $=360$ ), the figure provides equidistant slices through the spray distribution. Intensities are smoothed over $32^{3}$ voxels $\left(0.38^{3} \mathrm{~mm}^{3}\right)$ to reduce the granularity arising through the reconstruction of isolated single droplets.

The 3-d reconstruction gives a qualitative impression of the spatial spray distribution with intensity being a rough indicator of droplet size, since for an estimated resolution of $\chi>30 \mu \mathrm{m}$ the minimum intensity of the drop shadow image continuously decreases in the relevant drop size 
Fig. 19 Volumetric intensity distributions obtained with tomographic shadowgraph imaging at $p_{a}=4$ bar, $T_{a}=440 \mathrm{~K}, \mathrm{We}=360 ;$ Single shot smoothed over $32^{3}$ voxels (a) and average of 150 tomographic reconstructions (b)

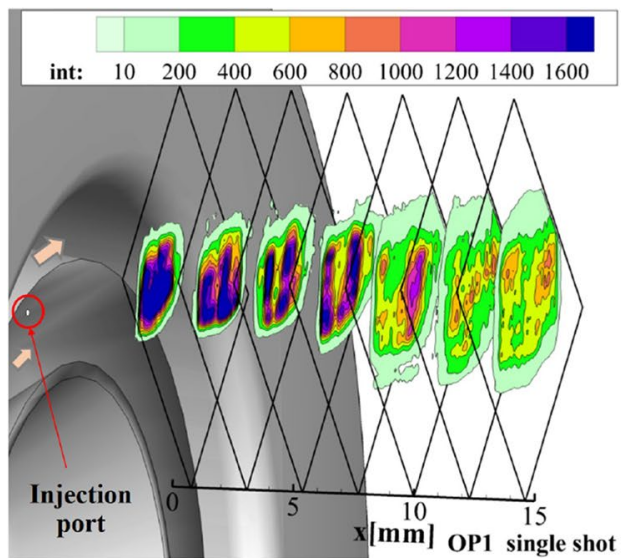

(a)

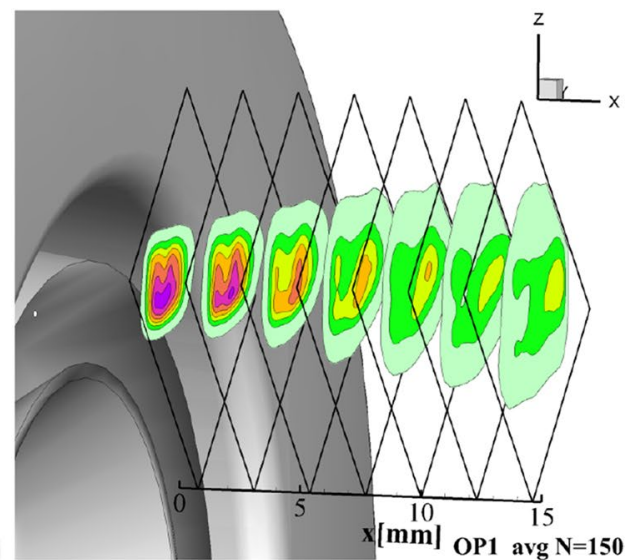

(b) range, as indicated in Fig. 13b, whereby the reconstruction is performed using inverted shadowgraphs (central intensity increases with drop size).

The average intensity distribution shown in Fig. 19b exhibits a u-shaped structure at $(x=1-8 \mathrm{~mm})$ and indicates regions where larger droplets appear more frequently. These regions seem to arise from the jet shear layer, where ligaments and drops are shed during surface break-up. Further downstream the windward leg of the $\mathrm{u}$-shaped structure disintegrates faster, possibly due to stronger interaction with the swirled flow. Prior to evaporation, larger droplets are carried radially outward in comparison to smaller droplets which follow the flow better. The u-shaped structure of the mean droplet distribution was also confirmed by visualizations of Mie-scattering using a planar light-sheet (Freitag 2018).

Figure 20 shows equidistant slices of the spray velocity field at increasing Weber number obtained from single shot results. Velocity data with correlation coefficients below $10 \%$ are blanked. The contour shape (axial velocity) can also be seen as a region, where droplets above the resolution limit of $d=10 \mu \mathrm{m}$ appear in coherent motion. The contour size and position can be used to track the spray trajectory and extension in space at fixed time (see also supplemental material). The size of the contoured area clearly decreases with rising air and fuel temperature indicating a significant droplet size reduction which is also evident in PDA measurements of liquid jet in cross flow atomization at elevated temperatures (cf. Freitag 2018).

Averaged spray velocities are shown in Fig. 21 and reveal slightly higher axial spray velocities on the windward side of the spray. Near the fuel injector face plate, both, instantaneous and time-averaged measurements show lower axial velocities inside the spray tail in comparison to outer regions. This might be due to the higher aerodynamic drag which the incoming air has to overcome until it reaches inner spray fragments, ligaments and droplets (Wu et al. 1998).
There are clear differences of the sizes of contoured areas between average (Fig. 21) and instantaneous results (Fig. 20) which indicates fluctuations of the spray tail position (e.g., flapping motion). These differences are more pronounced at $T_{a}=570 \mathrm{~K}$ and are also visible in the animations provided with the supplemental material.

At $x=10 \mathrm{~mm}$ downstream of the face plate, mean velocities are compared to 2-d PDA measurements acquired along the dashed line in Fig. 5 (Freitag 2018). Figure 22 shows axial (a) and tangential (b) velocity profiles of different droplet size classes in comparison to profiles obtained from 3-d correlation of two reconstructed volumes. Axial velocities are in agreement with velocities obtained from the $d=10 \mu \mathrm{m}$ and $d=15 \mu \mathrm{m}$ size classes except in regions with strong gradients (e.g., $y=7 \mathrm{~mm}$ ). Velocities obtained from 3-d correlation drop off near the edges, probably due to spatial averaging within the interrogation volume. Differences in tangential velocity are also present in regions with larger velocity gradients. Two possible explanations are: either velocities are smoothed due to the correlation over varying droplet sizes within one interrogation volume or there is an additional bias due to reconstruction ambiguities (so called 'ghost particles'). Moreover, compared to PDA, the interrogation volume size is considerably larger by factors of $10,3.6$ and 5 along $x, y$ and $z$, respectively. On the other hand, PDA measurements assume droplets to be spherical and are restricted to regions of reduced densities starting at about $10 \mathrm{~mm}$ downstream of the face plate.

\section{Discussion and perspectives}

A feasibility study was conducted on the 3-d reconstruction of spatial spray distributions under elevated pressure up to 7 bar and elevated temperature up to $570 \mathrm{~K}$ using simultaneous shadowgraph imaging from four views. Prediction of 3-c velocities of the liquid phase, that is, of droplets and 

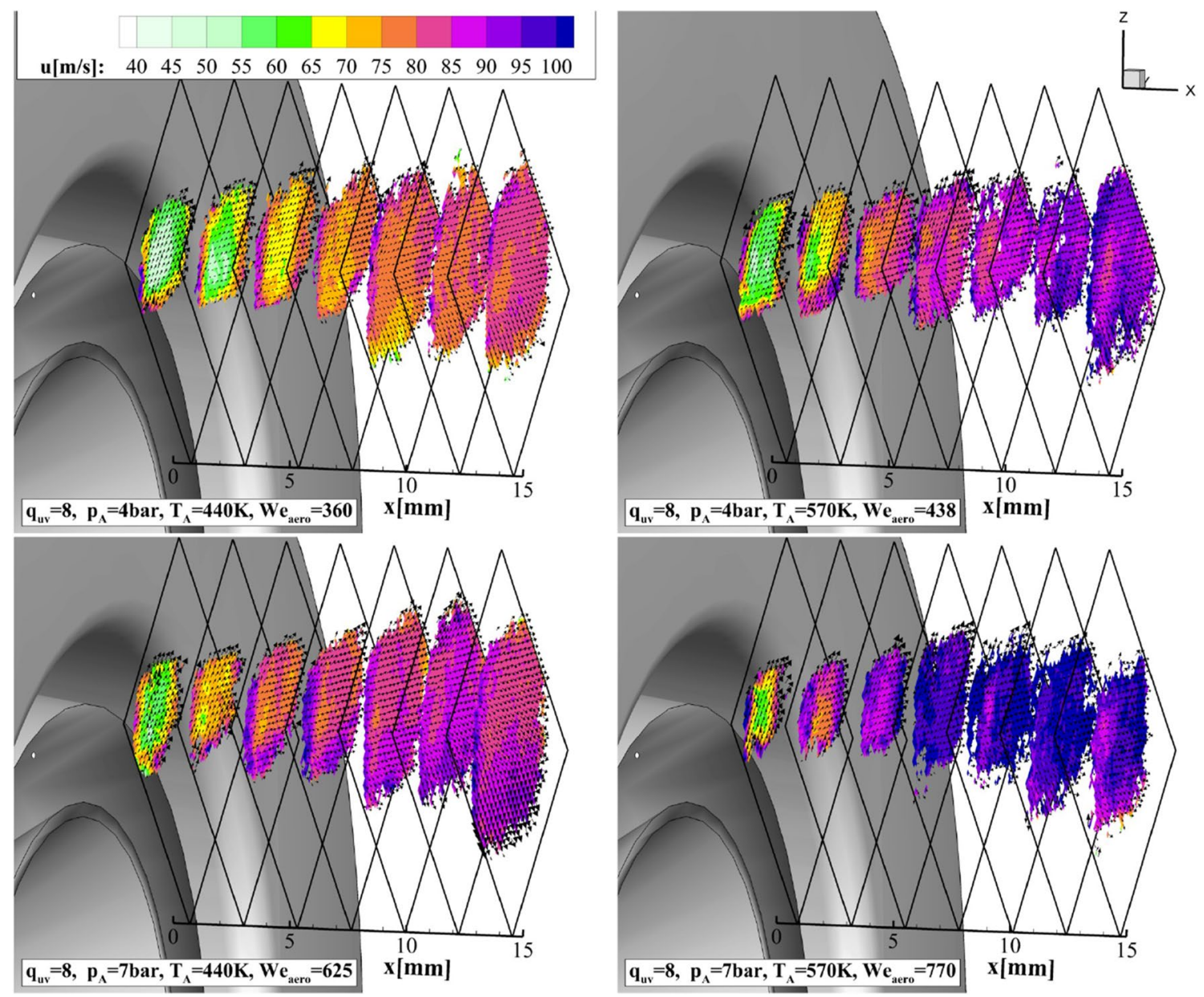

Fig. 20 Snap-shots of droplet velocities obtained from 3-d cross-correlation of single shots at different operation conditions (see Table 1). Vectors show the in-plane $V, W$ components. Animated versions of velocities are provided in the supplemental material

irregularly shaped ligaments, is based on cross-correlation processing of the reconstructed 3-d volumes. Thus, velocity fields provide estimates of the group velocity of the liquid phase inside each interrogation box which are not necessarily associated to the velocity of individual droplets. In principle, single droplet velocities can be obtained by subsequent tracking of each reconstructed droplet using matching techniques (e.g., nearest neighbour, neural network or relaxation techniques (Pereira et al. 2006)). Non-coherent image illumination relies on pulsed green LEDs, which on the one hand can deliver frame rates up to the $\mathrm{kHz}$ range at low cost and on the other hand provide temporally constant and spatially homogeneous illumination with sufficient signal strength at pulse widths in the $500 \mathrm{~ns}$ range and strongly stepped-down lenses.

Droplet streaking occurs due to the LED's finite response times and is a function of speed and droplet size which can lead to a reduction in contrast of the shadow images ( $10 \%$ elongation of the shadow of a $50 \mu \mathrm{m}$ droplet from $10 \mathrm{~m} / \mathrm{s}$ ) along with inaccuracies in the determination of the velocity using correlation analysis. This shortcoming can be overcome through a modification of the LED driver circuit originally presented by Willert et al. (2010) which potentially may reduce the response times to the 10-50 ns range (Halbritter et al. 2014; Kapustinsky et al. 1985).

The tomographically reconstructed 3-d intensity distribution from back-projected shadowgraphs gives a qualitative impression of the spray distribution with intensity being a rough indicator of droplet size. In principle, droplet sizes can be estimated, but this would require spatial calibration of the point spread function (PSF) and appropriate weighting of pixel intensities during 3-d reconstruction. The PSF was derived from the LSF which was measured on the basis of intensity gradients at edges of a lithographically produced checkerboard pattern. This procedure can be extended to spatially calibrate the PSF for each camera. Triangulation-based procedures of PSF evaluation (Schanz et al. 2013) require a homogeneous distributions of very 

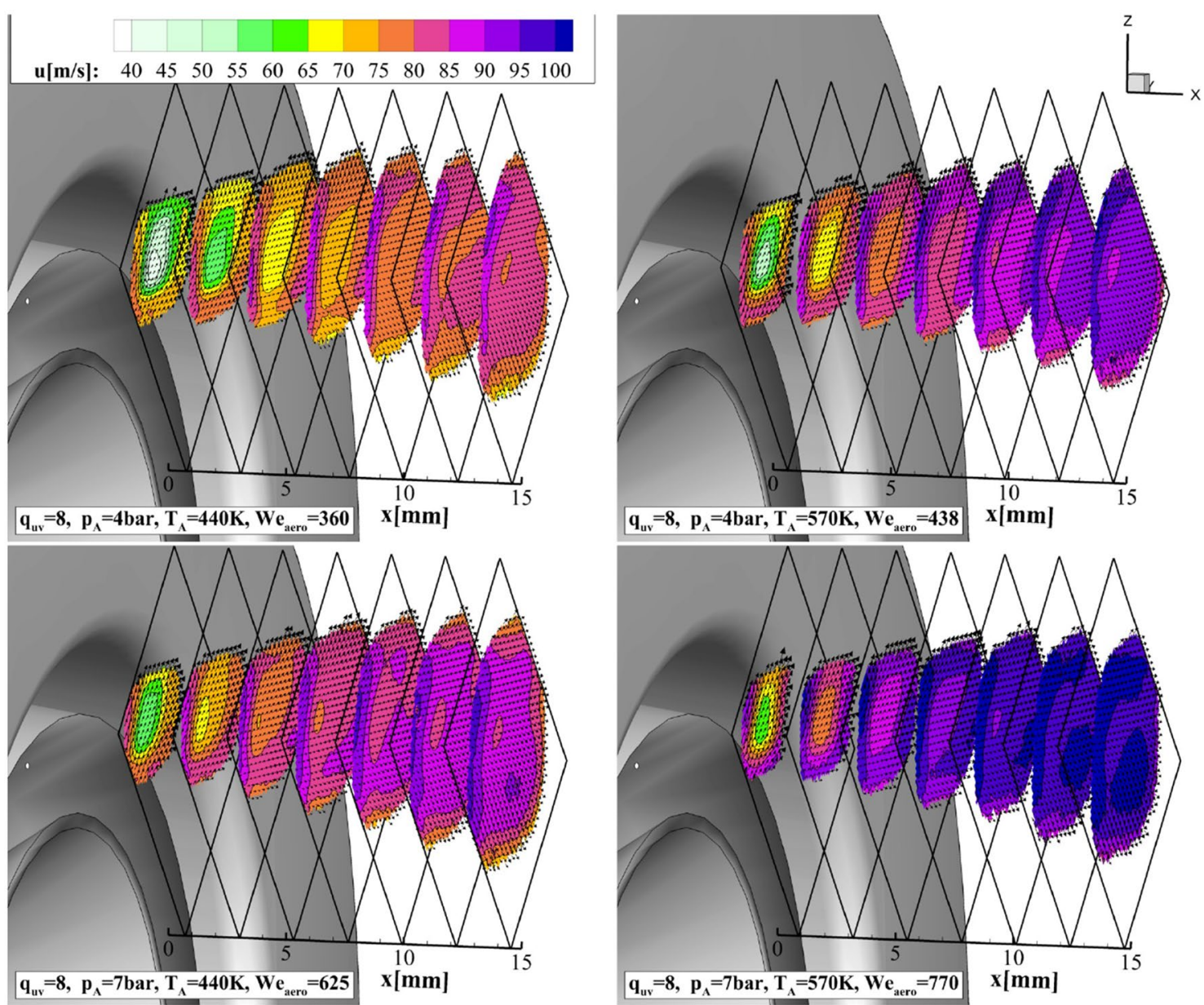

Fig. 21 Mean droplet velocities obtained by averaging 500 samples at different operation conditions (see Table 1). Vectors show the in-plane $V, W$ components

Fig. 22 Comparison of velocity profiles obtained by PDA and tomographic shadowgraph imaging (TS) at $x=10 \mathrm{~mm}$ aquired along the dotted line in Fig. 5 ( $v_{\mathrm{PDA}}$ runs parallel to the dotted line), $p_{a}=4 \mathrm{bar}$, $T_{a}=440 \mathrm{~K}, \mathrm{We}=360$

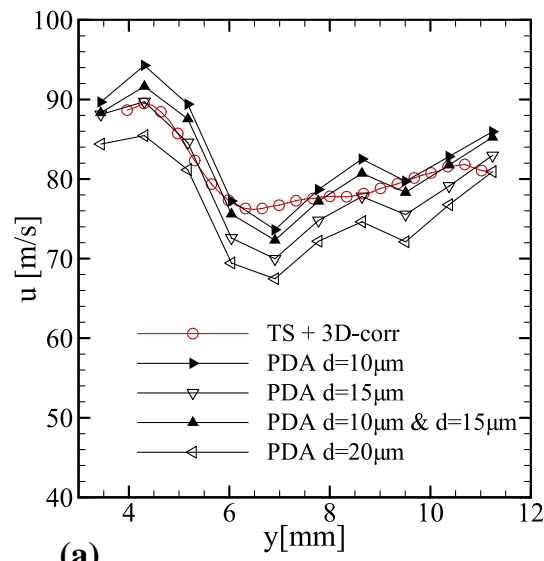

(a)

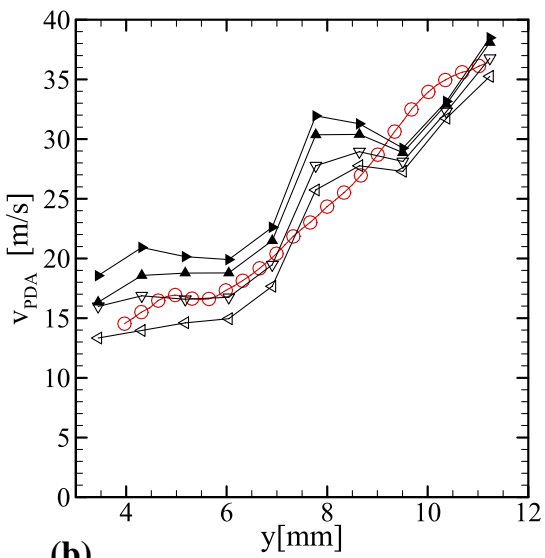

(b) small droplets as for PIV which is not feasible using inline LED illumination.

Issues introduced through the presence of ghost droplets arising from reconstruction ambiguities could be reduced significantly by using multi-pulse imaging along with Lagrangian tracking of individual droplets. Recently introduced algorithms such as the Shake-the-Box (STB) 3-d Lagrangian particle tracking method should be well suited for this purpose (Schanz et al. 2016) but would need modification to account for polydisperse droplet distributions. 
From a technical perspective, the high image magnification coupled with the high droplet velocities would require image acquisition rates in the $100 \mathrm{kHz}$ range which is still out of range for current mega-pixel sized high-speed imaging. A possible alternative could be to apply double frame imaging including double exposed shadowgraphs (multi-exposed recordings, Novara et al. (2019)) to create bursts of four time-instants for multi-pulse STB.

\section{Summary}

High-speed and tomographic shadowgraph imaging was successfully applied in a non-reactive kerosene spray in a pressurized environment with preheated swirled airflow mimicking the conditions of a fuel injector within an aero engine. The tomographic reconstruction of four views provided a common volume of $16 \times 13 \times 10 \mathrm{~mm}^{3}$ at a magnification close to unity using pulsed LED illumination. The use of compensator plates in the imaging path corrected for astigmatism induced by the thick windows of the pressure casing to obtain circular droplet images.

Estimations of the droplet shadow image contrast on the basis of the local point spread function revealed that the depth of focus strongly depends upon the droplet diameter. Under ideal conditions with the neglect of multi-scattering and streaking due to the finite pulse duration of the LEDs, the minimum resolvable droplet diameter of the described multiple view setup approaches $d=10 \mu \mathrm{m}$ within a depth of field of $-2 \mathrm{~mm}<z_{T S}<2 \mathrm{~mm}$ and increases to $d=20 \mu \mathrm{m}$ within the depth range $2 \mathrm{~mm} \leq\left|z_{T S}\right|<5 \mathrm{~mm}$. Velocity estimates of droplets immediately downstream of the fuel injector's exit plate were obtained by 3-d cross-correlation of two consecutive reconstructed droplet distributions using small interrogation volumes of $0.77 \times 0.38 \times 0.38 \mathrm{~mm}$ at a vector spacing of $0.38 \times 0.19 \times 0.19 \mathrm{~mm}(50 \%$ spatial overlap). Thereby, the three-dimensional velocity distribution of the instantaneous spray in a jet-in-cross-flow atomization configuration could be estimated at Weber numbers of We $=360-770$, air pressures of $p_{A}=4-7$ bar and air temperatures of $T_{A}=440-570 \mathrm{~K}$. Extracted slices of the instantaneous axial velocity indicate increased spatial motion (undulations) and fluctuations of the spray tail with increasing temperature.

The comparison to PDA velocity data showed good agreement for droplet size classes $d=10 \mu \mathrm{m}, 15 \mu \mathrm{m}$ but exhibited some deviations in regions with strong velocity gradients. These deviations are probably caused by spatial smoothing over droplets within the finite size of each interrogation volume and by the presence of reconstruction ambiguities ('ghost particles'). The latter shortcoming can be potentially improved by more advanced reconstruction techniques which use the local point spread function for volume sampling and image sampling instead of nearest neighbor techniques.

Acknowledgements The authors thank S. Freitag, I. Otterpohl und D. Döring for their support during the operation of the optical swirling spray injector test facility (OSSI) and for providing the PDA data sets.

Funding Open Access funding enabled and organized by Projekt DEAL.

\section{Declarations}

Conflict of Interest: The authors declare that they have no conflict of interest.

Code availability: The MENT-based volume reconstruction and hierarchical cross-correlation processing is based on DLR in-house codes.

Open Access This article is licensed under a Creative Commons Attribution 4.0 International License, which permits use, sharing, adaptation, distribution and reproduction in any medium or format, as long as you give appropriate credit to the original author(s) and the source, provide a link to the Creative Commons licence, and indicate if changes were made. The images or other third party material in this article are included in the article's Creative Commons licence, unless indicated otherwise in a credit line to the material. If material is not included in the article's Creative Commons licence and your intended use is not permitted by statutory regulation or exceeds the permitted use, you will need to obtain permission directly from the copyright holder. To view a copy of this licence, visit http://creativecommons.org/licenses/by/4.0/.

\section{References}

Astarita T, Cardone G (2005) Analysis of interpolation schemes for image deformation methods in PIV. Exp Fluids 38:233-243. https://doi.org/10.1007/s00348-004-0902-3

Becker J, Hassa C (2002) Breakup and atomization of a kerosene jet in crossflow at elevated pressure. At Sprays 12(1-3):49-67

Bilsky AV, Lozhkin VA, Markovich DM, Tokarev MP (2013) A maximum entropy reconstruction technique for tomographic particle image velocimetry. Meas Sci Technol 24(4):045301. https://doi. org/10.1088/0957-0233/24/4/045301

Blaisot JB, Yon J (2005) Droplet size and morphology characterization for dense sprays by image processing: application to the diesel spray. Exp Fluids 39(6):977-994. https://doi.org/10.1007/ s00348-005-0026-4

Braat J (1997) Analytical expressions for the wave-front aberration coefficients of a tilted plane-parallel plate. Appl Opt 36(32):8459 8467. https://doi.org/10.1364/AO.36.008459

Cai W, Powell CF, Yue Y, Narayanan S, Wang J, Tate MW, Renzi MJ, Ercan A, Fontes E, Gruner SM (2003) Quantitative analysis of highly transient fuel sprays by time-resolved X-radiography. Appl Phys Lett 83(8):1671-1673. https://doi.org/10.1063/1.1604161

Cao L, Pan G, de Jong J, Woodward S, Meng H (2008) Hybrid digital holographic imaging system for three-dimensional dense particle field measurement. Appl Opt 47(25):4501-4508. https://doi.org/ 10.1364/AO.47.004501

Claxton CD, Staunton RC (2008) Measurement of the point-spread function of a noisy imaging system. J Opt Soc Am A 25(1):159170. https://doi.org/10.1364/JOSAA.25.000159 
Coletti F, Benson MJ, Sagues AL, Miller BH, Fahrig R, Eaton JK (2014) Three-dimensional mass fraction distribution of a spray measured by x-ray computed tomography. J Eng Gas Turbines Power 136(5):051508-051508-8. https://doi.org/10.1115/1.40262 45

Elsinga G, Scarano F, Wieneke B, van Oudheusden B (2006) Tomographic particle image velocimetry. Exp Fluids 41:933-947

Fansler TD, Parrish SE (2015) Spray measurement technology: a review. Meas Sci Technol 26(1):012002. https://doi.org/10.1088/ 0957-0233/26/1/012002

Fdida N, Blaisot JB (2010) Drop size distribution measured by imaging: determination of the measurement volume by the calibration of the point spread function. Meas Sci Technol 21(2):025501

Freitag S (2016) Experimentelle Untersuchung der Kraftstoffaufbereitung in einer verdrallten Luftströmung unter realistischen Drücken und Vorwärmtemperaturen ohne Reaktion in einem Einzelsektor-Brennkammermodell mit punktuellem Kraftstoffeintrag. In: Deutscher Luft- und Raumfahrtkongress, 13.-15. September, Braunschweig

Freitag S (2018) Experimental investigations of fuel preparation in a swirling airflow under realistic conditions without reaction in a combustor model with a point fuel source. CEAS Aeronaut $\mathrm{J}$ 9:475-490. https://doi.org/10.1007/s13272-018-0295-2

Freitag S (2019) Untersuchung eines punktuellen Kraftstoffeintrags in einem Brennkammermodell unter realistischen Betriebsbedingungen ohne Verbrennung. PhD thesis, Ruhr-Universität Bochum, Bochum,

Ganapathisubramani B, Clemens NT (2006) Effect of laser pulse duration on particle image velocimetry. AIAA J. https://doi.org/10. 2514/1.18404

Giskes E, Verschoof RA, Segerink FB, Venner CH (2016) Schlieren study of a sonic jet injected into a supersonic cross flow using high-current pulsed LEDs. 69th Annual Meeting of the APS Division of Fluid Dynamics - Gallery of Fluid Motion https://doi.org/ 10.1103/aps.dfd.2016.gfm.v0067, arXiv:1606.06683

Guildenbecher DR, Wagner JL, Olles JD, Demauro EP, Farias P, Grasser TW, Sojka PE (2016) kHz rate digital in-line holography applied to quantify secondary droplets from the aerodynamic breakup of a liquid column in a shock-tube. In: 54th AIAA Aerospace Sciences Meeting, 4-8 January 2016, San Diego, California, USA, https://doi.org/10.2514/6.2016-1044,

Halbritter H, Jäger C, Weber R, Schwind M, Möllmer F (2014) Highspeed LED driver for ns-pulse switching of high-current LEDs. IEEE Photonics Technol Lett 26(18):1871-1873. https://doi.org/ 10.1109/LPT.2014.2336732

Halls B, Radke C, Heindel T, Lohry W, Zhang S, Meyer T, Lightfoot M, Danczyk S, Schumaker S, Roy S, Gord J, Kastengren A (2013) Characterization of three-dimensional dense spray visualization techniques. In: 51st AIAA Aerospace Sciences Meeting including the New Horizons Forum and Aerospace Exposition, https://doi. org/10.2514/6.2013-477,

Halls BR, Rahman N, Slipchenko MN, James JW, McMaster A, Ligthfoot MDA, Gord JR, Meyer TR (2019) 4d spatiotemporal evolution of liquid spray using kilohertz-rate $\mathrm{x}$-ray computed tomography. Opt Lett 44(20):5013-5016. https://doi.org/10.1364/OL. 44.005013

Joint Committee for Guides in Metrology (JCGM) (2008) Evaluation of measurement data - guide to the expression of uncertainty in measurement GUM, JCGM 100:2008. http://www.bipm.org/en/ publications/guides/gum.html

Jones AR, Sarjeant M, Davis CR, Denham RO (1978) Application of in-line holography to drop size measurement in dense fuel sprays. Appl Opt 17(3):328-330

Jones RC (1958) On the point and line spread functions of photographic images. J Opt Soc Am 48(12):934-937. https://doi.org/ 10.1364/JOSA.48.000934
Kang B, Poulikakos D (1996) Holography experiments in a dense highspeed impinging jet spray. J Propuls Power 12(2):341-348

Kapustinsky JS, DeVries R, DiGiacomo N, Sondheim W, Sunier J, Coombes H (1985) A fast timing light pulser for scintillation detectors. Nucl Instrum Methods Phys Res A241:612-613. https:// doi.org/10.1016/0168-9002(85)90622-9

Klinner J (2017) Development and assessment of volume resolving velocimetry for turbomachinery test facilities. PhD thesis, University of Kassel, https://elib.dlr.de/117111/

Klinner J, Willert C (2012) Tomographic shadowgraphy for threedimensional reconstruction of instantaneous spray distributions. Exp Fluids 53(2):531-543. https://doi.org/10.1007/ s00348-012-1308-2

Kourmatzis A, Pham PX, Masri AR (2017) A two-angle far-field microscope imaging technique for spray flows. Meas Sci Technol 28(3):035302. https://doi.org/10.1088/1361-6501/aa5525

Laven P (2003) Simulation of rainbows, coronas, and glories by use of mie theory. Appl Opt 42(3):436-444. https://doi.org/10.1364/ AO.42.000436

Liu X, Im KS, Wang Y, Wang J, Tate MW, Ercan A, Schuette DR, Gruner SM (2009) Four dimensional visualization of highly transient fuel sprays by microsecond quantitative $\mathrm{x}$-ray tomography. Appl Phys Lett 94(8):084101. https://doi.org/10.1063/1. 3048563

Lü Q, Chen Y, Yuan R, Ge B, Gao Y, Zhang Y (2009) Trajectory and velocity measurement of a particle in spray by digital holography. Appl Opt 48(36):7000-7007. https://doi.org/10.1364/AO. 48.007000

Meng H, Hussain F (1995) In-line recording and off-axis viewing technique for holographic particle velocimetry. Appl Opt 34(11):1827-1840. https://doi.org/10.1364/AO.34.001827

Nasibov H, Balaban E, Kholmatov A, Nasibov A (2014) High-brightness, high-power led-based strobe illumination for double-frame micro particle image velocimetry. Flow Meas Instrum 37:12-28. https://doi.org/10.1016/j.flowmeasinst.2014.03.004

Novara M, Schanz D, Geisler R, Gesemann S, Voss C, Schröder A (2019) Multi-exposed recordings for 3D lagrangian particle tracking with multi-pulse shake-the-box. Exp Fluids 60(3):44. https://doi.org/10.1007/s00348-019-2692-7

Pereira F, Stüer H, Graff EC, Gharib M (2006) Two-frame 3d particle tracking. Meas Sci Technol 17(7):1680. https://doi.org/10.1088/ 0957-0233/17/7/006

Pham PX, Kourmatzis A, Masri AR (2017) Simultaneous volumevelocity measurements in the near field of atomizing sprays. Meas Sci Technol 28(11):115203. https://doi.org/10.1088/13616501/aa8893

Rachner M (1998) Die Stoffeigenschaften von Kerosin Jet A-1. Tech. Rep. ISRN DLR-FB-98-01, DLR

Raffel M, Willert C, Scarano F, Kähler C, Wereley S, Kompenhans J (2017) Particle image velocimetry. Exp Fluid Mech, Springer Int Publ AG,. https://doi.org/10.1007/978-3-319-68852-7

Rao UVG, Jain VK (1967) Gaussian and exponential approximations of the modulation transfer function. J Opt Soc Am 57(9):1159 1160. https://doi.org/10.1364/JOSA.57.001159

Santangelo PJ, Sojka PE (1994) Focused-image holography as a dense-spray diagnostic. Appl Opt 33(19):4132-4136. https:// doi.org/10.1364/AO.33.004132

Scarano F (2002) Iterative image deformation methods in PIV. Meas Sci Technol 13(1):R1, http://stacks.iop.org/0957-0233/13/i=1/ $\mathrm{a}=201$

Schanz D, Gesemann S, Schröder A, Wieneke B, Novara M (2013) Non-uniform optical transfer functions in particle imaging: calibration and application to tomographic reconstruction. Meas Sci Technol 24(2):024009. https://doi.org/10.1088/0957-0233/ 24/2/024009 
Schanz D, Gesemann S, Schröder A (2016) Shake-The-Box: Lagrangian particle tracking at high particle image densities. Exp Fluids 57(5):70. https://doi.org/10.1007/s00348-016-2157-1

Soria J (1998) Multigrid approach to cross-correlation digital PIV and HPIV analysis. In: Proceedings of the Thirteenth Australasian Fluid Mechanics Conference, Melbourne, Australia, 13-18 December 1998, pp 381-384, https://www.scopus.com/inward/ record.uri?eid=2-s2.0-0032232442\& partnerID $=40 \& \mathrm{md} 5=$ 61d40aca10dc0ff 235ef520c4a083042, cited By 40

Soria J, Atkinson C (2008) Towards 3C-3D digital holographic fluid velocity vector field measurement - tomographic digital holographic PIV (Tomo-HPIV). Meas Sci Technol 19(7):074002. https://doi.org/10.1088/0957-0233/19/7/074002

Thévenaz P, Blu T, Unser M (2000) Interpolation revisited. IEEE Trans Med Imaging 19(7):739-758

Westerweel J (2000) Theoretical analysis of the measurement precision in particle image velocimetry. Exp Fluids 29:S003-S012. https://doi.org/10.1007/s003480070002

Westerweel J, Scarano F (2005) Universal outlier detection for PIV data. Exp Fluids 39(6):1096-1100. https://doi.org/10.1007/ s00348-005-0016-6

Willert C, Stasicki B, Klinner J, Moessner S (2010) Pulsed operation of high-power light emitting diodes for imaging flow velocimetry. Meas Sci Technol 21(7):1-12. https://doi.org/10.1088/0957-0233/ 21/7/075402
Willert C, Mitchell D, Soria J (2012) An assessment of high-power light-emitting diodes for high frame rate schlieren imaging. Exp Fluids 53:413-421. https://doi.org/10.1007/s00348-012-1297-1

Wu PK, Kirkendall KA, Fuller RP, Nejad AS (1998) Spray structures of liquid jets atomized in subsonic crossflows. J Propuls Power 14(2):173-182. https://doi.org/10.2514/2.5283

Wu X, Lin W, Wang L, Song G, Wu Y (2021) Measurement of airblast atomization of low temperature kerosene with $25 \mathrm{khz}$ digital holography. Appl Opt 60(4):A131-A139. https://doi.org/10.1364/ AO.404322

Yang Y, Kang B (2009) Measurements of the characteristics of spray droplets using in-line digital particle holography. J Mech Sci Technol 23:1670-1679. https://doi.org/10.1007/s12206-009-0417-1

Zheng Q, Qin T, Li W, Wang X, Huang Y, Liu Z (2021) Astigmatism analysis and correction method introduced by an inclined plate in a convergent optical path. Appl Opt 60(4):875. https://doi.org/ 10.1364/AO.412997

Publisher's Note Springer Nature remains neutral with regard to jurisdictional claims in published maps and institutional affiliations. 